\title{
The Global and Exponential Attractors for the Higher-order Kirchhoff-type Equation with Strong Linear Damping
}

\author{
Guoguang $\operatorname{Lin}^{1}$, Yunlong $\mathrm{Gao}^{2}$ \\ ${ }^{1}$ Department of Mathematics, Yunnan University, Kunming, Yunnan 650091, People's Republic of China \\ 2 Department of Mathematics, Yunnan University, Kunming, Yunnan 650091, People’s Republic of China \\ Correspondence: Department of Mathematics, Yunnan University, Kunming, Yunnan 650091, \\ People's Republic of China. E-mail: gglin@ynu.edu.cn
}

Received: May 31, 2017 Accepted: June 21, 2017 Online Published: July 24, 2017

doi:10.5539/jmr.v9n4p145 URL: https://doi.org/10.5539/jmr.v9n4p145

This work is supported by the National Natural Sciences Foundation of People's Republic of China under Grant 11561076

\begin{abstract}
In this paper, we study the longtime behavior of solution to the initial boundary value problem for a class of strongly damped Higher-order Kirchhoff type equations: $u_{t t}+(-\Delta)^{m} u_{t}+\left(\alpha+\beta\left\|\nabla^{m} u\right\|^{2}\right)^{q}(-\Delta)^{m} u+g(u)=f(x)$. At first, we do priori estimation for the equations to obtain two lemmas and prove the existence and uniqueness of the solution by the lemmas and the Galerkin method. Then, we obtain to the existence of the global attractor in $H_{0}^{m}(\Omega) \times L^{2}(\Omega)$ according to some of the attractor theorem. In this case, we consider that the estimation of the upper bounds of Hausdorff for the global attractors are obtained. At last, we also establish the existence of a fractal exponential attractor with the non-supercritical and critical cases.
\end{abstract}

Keywords: Nonlinear Higher-order Kirchhoff type equation, Galerkin method, The existence and uniqueness, The Global attractor, Huasdorff dimensions, The Exponential attractor

2010 Mathematics Classification: 35K10, 35K10, 35K41

\section{Introduction}

In this paper, we are concerned with the existence of global attractor for the following nonlinear Higher-order Kirchhofftype equations:

$$
\begin{aligned}
& u_{t t}+(-\Delta)^{m} u_{t}+\left(\alpha+\beta\left\|\nabla^{m} u\right\|^{2}\right)^{q}(-\Delta)^{m} u+g(u)=f(x),(x, t) \in \Omega \times[0,+\infty), \\
& u(x, 0)=u_{0}(x), u_{t}(x, 0)=u_{1}(x), x \in \Omega, \\
& u(x, t)=0, \frac{\partial^{i} u}{\partial v^{i}}=0, i=1, \ldots, m-1, x \in \partial \Omega, t \in(0,+\infty),
\end{aligned}
$$

where $\mathrm{m}>1$ is an integer constant, $\alpha>0, \beta>0$ are constants and $q$ is a real number. Moreover, $\Omega$ is a bounded domain in $R^{n}$ with the smooth boundary $\partial \Omega$ and $v$ is the unit outward normal on $\partial \Omega . g(u)$ is a nonlinear function specified later.

It is known that Kirchhoff (1883) first investigated the following nonlinear vibration of an elastic string for $\delta=f=0$ :

$$
\rho h \frac{\partial^{2} u}{\partial t^{2}}+\delta \frac{\partial u}{\partial t}=\left\{p_{0}+\frac{E h}{2 L} \int_{0}^{L}\left(\frac{\partial u}{\partial x}\right)^{2} d x\right\} \frac{\partial^{2} u}{\partial x^{2}}+f ; \quad 0 \leqslant x \leqslant L, t \geqslant 0,
$$

where $u=u(x, t)$ is the lateral displacement at the space coordinate $x$ and the time $t, \rho$ the mass density, $h$ the cross-section area, $L$ the length, $E$ the Young modulus, $p 0$ the initial axial tension, $\delta$ the resistance modulus, and $f$ the external force.

When $\alpha=0, \beta=1$ and $q>0$ are real number, Yunlong Gao, Yuting Sun and Guoguang Lin (2016) studied existence of weak solutions for degenerate High-order Kirchhoff equations:

$$
\begin{aligned}
& u_{t t}+(-\Delta)^{m} u_{t}+\left\|\nabla^{m} u\right\|^{2 q}(-\Delta)^{m} u+g(u)=f(x),(x, t) \in \Omega \times[0,+\infty), \\
& u(x, 0)=u_{0}(x), u_{t}(x, 0)=u_{1}(x), x \in \Omega, \\
& u(x, t)=0, \frac{\partial^{i} u}{\partial v^{i}}=0, i=1, \ldots, m-1, x \in \partial \Omega, t \in(0,+\infty),
\end{aligned}
$$


where $\mathrm{m}>1$ is an integer constant. $\Omega$ is a bounded domain in $R^{n}$ with the smooth boundary $\partial \Omega$ and $v$ is the unit outward normal on $\partial \Omega . g(u)$ is a nonlinear function specified later.

When $\alpha=0, \beta=1$ and $q>0$ is real number and strong linear stamping $(-\Delta)^{m} u_{t}$ is replaced $\beta u_{t}$, Li Yan (2011) studied The Asymptotic Behavior of Solutions for a Nonlinear Higher Order Kirchhoff Type Equation:

$$
\begin{aligned}
& u_{t t}+\left(\int_{\Omega}\left|D^{m} u\right|^{2} d x\right)^{q}(-\Delta)^{m} u+\beta u_{t}+g(u)=0, \text { in } \quad Q=\Omega \times(0,+\infty), \\
& u(x, t)=0, \frac{\partial^{i} u}{\partial v^{i}}=0, i=1,2, \ldots, m-1, \text { on } \quad \sum=\Gamma \times(0,+\infty), \\
& u(x, 0)=u_{0}(x), u_{t}(x, 0)=u_{1}(x), \text { in } \quad x \in \Omega,
\end{aligned}
$$

where $\Omega$ is an open bounded set of $R^{n}(n \geq 1)$ with smooth boundary $\Gamma$ and the unit normal vector.The function $g \in C^{1}$ satisfies some of conditions.

When $\left(\alpha+\beta\left\|\nabla^{m} u\right\|^{2}\right)^{q}$ is replaced $a+b\left\|\nabla^{m} u\right\|^{2 q}$ and $g(u)=-|u|^{p} u$, Guoguang Lin, Yunlong Gao, Yuting Sun (2017) had studied local existence and blow-up of solutions:

$$
\begin{aligned}
& u_{t t}+(-\Delta)^{m} u_{t}+\left(a+b\left\|D^{m} u\right\|^{2 q}\right)(-\Delta)^{m} u=|u|^{p} u,(x, t) \in \Omega \times[0,+\infty), \\
& u(x, t)=0, \frac{\partial^{i} u}{\partial v^{i}}=0, i=1,2, \cdots, m-1, x \in \partial \Omega, t \in(0,+\infty), \\
& u(x, 0)=u_{0}(x), u_{t}(x, 0)=u_{1}(x), x \in \Omega,
\end{aligned}
$$

where $\Omega$ is a bounded domain in $R^{n}$ with the smooth boundary $\partial \Omega$ and $v$ is the unit outward normal on $\partial \Omega$. Moreover, $m>1$ is an integer constant, and $q, p, a$ and $b$ are some constants such that $q \geq 1, p \geq 0, a \geq 0, b \geq 0$ and $a+b>0$.

When $q=0, m=1, g(u)=-|u|^{p} u$, the equation (1.1) becomes a nonlinear wave equation:

$$
\begin{aligned}
& u_{t t}-\Delta u-\Delta u_{t}=|u|^{p} u, \quad(x, t) \in \Omega \times[0,+\infty), \\
& u(x, 0)=u_{0}(x), u_{t}(x, 0)=u_{1}(x), \quad x \in \Omega, \\
& u(x, t)=0, \quad(x, t) \in \partial \Omega \times[0,+\infty) .
\end{aligned}
$$

It has been extensively studied and several results concerning existence and blowing-up have been established (Ball, J. M., 1997; KOPÁC̆KOVÁ, M, 1989; HARAUX, A. \& ZUAZUA, E., 1988).

When $\alpha=0, \beta=1, m=1, g(u)=-|u|^{\alpha} u$ and $q=\gamma>0$ is real number, Kosuke Ono (1997) had studied global existence, asymptotic stability and blowing up of solutions for Some Degenerate Non-linear Wave Equations:

$$
\begin{aligned}
& u_{t t}-\|\nabla u\|^{2 \gamma} \Delta u-\Delta u_{t}=|u|^{\alpha} u, \quad(x, t) \in \Omega \times[0,+\infty), \\
& u(0)=u_{0}(x), u_{t}(0)=u_{1}(x), \quad x \in \Omega, \\
& \left.u(x, t)\right|_{\partial \Omega}=0, \quad t \in[0,+\infty),
\end{aligned}
$$

where $\Omega$ is a bounded domain in $R^{n}$ with the smooth boundary $\partial \Omega$.

When $\left(\alpha+\beta\left\|\nabla^{m} u\right\|^{2}\right)^{q}$ is replaced $-m\left(\int_{\Omega}|\nabla u(t, x)|^{2} d x\right), m=1, g(u)=0$ and no linear damping, Marina Ghisi and Massimo Gobbino (2009) studied spectral gap global solutions for degenerate Kirchhoff equations. Given a continuous function $m:[0,+\infty) \rightarrow[0,+\infty)$, they consider the Cauchy problem:

$$
\begin{aligned}
& u_{t t}(t, x)+m\left(\int_{\Omega}|\nabla u(t, x)|^{2} d x\right) \Delta u(t, x)=0, \forall(x, t) \in \Omega \times[0, T), \\
& u(0)=u_{0}, u_{t}(0)=u_{1},
\end{aligned}
$$

where $\Omega \subseteq R^{n}$ is an open set and $\nabla u$ and $\Delta u$ denote the gradient and the Laplacian of $\mathrm{u}$ with respect to the space variables. They prove that for such initial data $\left(u_{0}, u_{1}\right)$ there exist two pairs of initial data $\left(\bar{u}_{0}, \bar{u}_{1}\right),\left(\hat{u}_{0}, \hat{u}_{1}\right)$ for which the solution is global, and such that $u_{0}=\bar{u}_{0}+\hat{u}_{0}, u_{1}=\bar{u}_{1}+\hat{u}_{1}$.

When $m=1,\left(\alpha+\beta\left\|\nabla^{m} u\right\|^{2}\right)^{q}$ and $(-\Delta)^{\alpha} u_{t}$ are replaced $M\left(\|\nabla u\|^{2}\right),(-\Delta) u_{t}$. Yang Zhijian, Ding Pengyan and Lei Li (2016) studied Longtime dynamics of the Kirchhoff equations with fractional damping and supercritical nonlinearity:

$$
u_{t t}-M\left(\|\nabla u\|^{2}\right) \Delta u+(-\Delta)^{\alpha} u_{t}+f(u)=g(x), x \in \Omega, t>0,
$$




$$
\left.u\right|_{\partial \Omega}=0, u(x, 0)=u_{0}(x), u_{t}(x, 0)=u_{1}(x)
$$

where $\alpha \in\left(\frac{1}{2}, 1\right), \Omega$ is a bounded domain $R_{N}$ with the smooth boundary $\partial \Omega$, and the nonlinearity $f(u)$ and external force term $g$ will be specified.The main results are focused on the relationships among the growth exponent p of the nonlinearity $f(u)$ and well-posedness. They show that (i)even if $\mathrm{p}$ is up to the supercritical range, that is, $1 \leq p<\frac{N+4 \alpha}{(N-4 \alpha)^{+}}$, the wellposedness and the longtime behavior of the solutions of the equation are of the characters of the parabolic equation; (ii) when $\frac{N+4 \alpha}{(N-4 \alpha)^{+}} \leq p<\frac{N+4}{(N-4)^{+}}$, the corresponding subclass G of the limit solutions exists and possesses a weak global attractor.

When $m=1,\left(\alpha+\beta\left\|\nabla^{m} u\right\|^{2}\right)^{q}$ is replaced $\sigma\left(\|\Delta u\|^{2}\right)$, Yang Zhijian, I.Chueshov ( Yang, Z. J. \& et al., 2014; Zhijian Yang \& Zhiming Liu., 2015; Igor Chueshov., 2012) studied the Global attractor and exponential attractors for the Kirchhoff type equations with strong nonlinear damping and supercritical nonlinearity:

$$
\begin{aligned}
& u_{t t}-\sigma\left(\|\Delta u\|^{2}\right) \Delta u_{t}-\phi\left(\|\Delta u\|^{2}\right) \Delta u+f(u)=h(x) \text { in } \Omega \times \mathbb{R}^{+}, \\
& \left.u(x, t)\right|_{\partial \Omega}=0, u(x, 0)=u_{0}(x), u_{t}(x, 0)=u_{1}(x), \quad x \in \Omega .
\end{aligned}
$$

where $\Omega$ is a bounded domain in $R^{N}$ with the smooth boundary $\partial \Omega, \sigma(s), \phi(s)$ and $f(s)$ are nonlinear functions, and $h(x)$ is an external force term. They prove that in strictly positive stiffness factors and supercritical nonlinearity case, there exists a global finite-dimensional attractor in the natural energy space endowed with strong topology.

When $m=1$, Xiaoming Fan (2004) consider the following non-degenerate Kirchhoff-type's Kernel sections and estimation of Hausdorff dimensions:

$$
\begin{aligned}
& u_{t t}-\alpha \Delta u_{t}-\left(\beta+\gamma\left(\int_{\Omega}|\nabla u|^{2} d x\right)^{\rho}\right) \Delta u+h\left(u_{t}\right)+f(u, t)=g(x, t), x \in \Omega, t>\tau, \\
& \left.u(x, t)\right|_{x \in \partial \Omega}=0, t \geq \tau \\
& u(x, \tau)=u_{0 \tau}(x), u_{t}(x, t)=u_{1 \tau}(x), x \in \Omega,
\end{aligned}
$$

where $\beta>0, \rho>-1, \gamma \geq 0 . h\left(u_{t}\right)$ and $g(u, t)$ are supposed in paper.

For the most of the scholars represented by Yang Zhijian have studied all kinds of low order Kirchhoff equations and only a small number of scholars have studied the blow-up and asymptotic behavior of solutions for higher-order Kirchhoff equation. So, in this context, we study the high-order Kirchhoff equation is very meaningful. In order to study the high-order nonlinear Kirchhoff equation with the damping term, we borrow some of Li Yan's (Ball, J. M., 1997) partial assumptions (2.1)-(2.3) for the nonlinear term $\mathrm{g}$ in the equation. In order to prove that the lemma 2.4, we have improved the results from assumptions (2.1)-(2.3) such that $0<C_{2} \leq \frac{1}{2}$. Then, under all assumptions, we prove that the equation has a unique smooth solution $\left(u, u_{t}\right) \in L^{\infty}\left((0,+\infty) ; H^{2 m}(\Omega) \cap H_{0}^{m}(\Omega) \times H_{0}^{m}(\Omega)\right)$ and obtain the solution semigroup $S(t)$ : $H^{2 m}(\Omega) \cap H_{0}^{m}(\Omega) \times H_{0}^{m}(\Omega) \rightarrow H^{2 m}(\Omega) \cap H_{0}^{m}(\Omega) \times H_{0}^{m}(\Omega)$ has global attractor $\mathcal{A}$ and the upper bounds of Hausdorff dimensions. At last, we get the exponential attractor by strong quasi-stability.

For more related results we refer the reader to (Xiaoming Fan \& Shengfan Zhou., 2004; HD Nguyen., 2014; Yaojun Ye, 2013; Teman, R., 1998; S. Zhou., 1999; Ke Li., 2017; Zhang Yan \& et al., 2008; Xueli Song \& Yanren Hou., 2015; L. H. Fatori, et al, 2015; Lin, G. G., 2011; Teman, R., 1998; Wu, J. Z. \& Lin, G. G., 2009; Robert A. Adams, et al., 2003 ; Z. J. Yang, 2010; Zhijian Yang \& Pengyan Ding, 2016). In order to make these equations more normal, in section 2 and in section 3, some assumptions, notations and the main results are stated. Under these assumptions, we prove the existence and uniqueness of solution, then we obtain the global attractors for the problems (1.1)-(1.3). In section 4, we consider that the estimation of the upper bounds of Hausdorff for the global attractors are obtained according to (Yaojun Ye., 2013). In section 5, we obtain the fractal exponential attractor by (Yang, Z. J. \& et al., 2016; Yang, Z. J. \& et al., 2014; Zhijian Yang \& Zhiming Liu, 2015; Igor Chueshov, 2012).

\section{Preliminaries}

In this section, we introduce material needed in the proof our main result. We use the standard Lebesgue space $L^{p}(\Omega)$ and Sobolev space $H^{m}(\Omega)$ with their usual scalar products and norms. Meanwhile we define $H_{0}^{m}(\Omega)=\left\{u \in H^{m}(\Omega): \frac{\partial^{i} u}{\partial v^{i}}=0, i=0,1, \ldots, m-1\right\}$ and introduce the following abbreviations: $E_{0}=H_{0}^{m}(\Omega) \times L^{2}(\Omega), E_{1}=$ $H^{2 m}(\Omega) \cap H_{0}^{m}(\Omega) \times H_{0}^{m}(\Omega), A=-\Delta,\|\cdot\|_{H^{m}}=\|\cdot\|_{H^{m}(\Omega)},\|\cdot\|_{H_{0}^{m}}=\|\cdot\|_{H_{0}^{m}(\Omega)},\|\cdot\|=\|\cdot\|_{L^{2}(\Omega)},\|\cdot\|_{p}=\|\cdot\|_{L^{p}(\Omega)}$ for any real number $p>1$.

According to (Li, Y., 2011), we present some assumptions and notations needed in the proof of our results. For this reason, we assume nonlinear term $g(u) \in C^{1}(\Omega)$ satisfies that 
$\left(H_{1}\right)$ Setting $G(s)=\int_{0}^{s} g(r) d r$, then

$$
\lim _{|s| \rightarrow \infty} \inf \frac{G(s)}{s^{2}} \geq 0
$$

$\left(H_{2}\right)$ If

$$
\lim _{|s| \rightarrow \infty} \sup \frac{\left|g^{\prime}(s)\right|}{|s|^{r}}=0,
$$

where $0 \leq r<+\infty(n=1,2), 0 \leq r<2(n=3), r=0(n \geq 4)$.

$\left(H_{3}\right)$ There exist constant $C_{0}>0$, such that

$$
\lim _{|s| \rightarrow \infty} \inf \frac{s g(s)-C_{0} G(s)}{s^{2}} \geq 0 .
$$

$\left(H_{4}\right)$ There exist constant $C_{1}>0$, such that

$$
\begin{aligned}
& |g(s)| \leq C_{1}\left(1+|s|^{p}\right), \\
& \left|g^{\prime}(s)\right| \leq C_{1}\left(1+|s|^{p-1}\right),
\end{aligned}
$$

where $1 \leq p \leq \frac{n+2 m}{n-2 m}(n>2 m)$ and $1 \leq p<+\infty(n \leq 2 m)$.

For every $\gamma>0$, by $\left(H_{1}\right)-\left(H_{3}\right)$ and apply Poincaré inequality, there exist constants $C(\gamma)>0$, such that

$$
\begin{aligned}
& J(u)+\gamma\left\|\nabla^{m} u\right\|^{2}+C(\gamma) \geq 0, \quad \forall u \in H^{m}(\Omega), \\
& (g(u), u)-C_{2} J(u)+\gamma\left\|\nabla^{m} u\right\|^{2}+C(\gamma) \geq 0, \quad \forall u \in H^{m}(\Omega),
\end{aligned}
$$

where $J(u)=\int_{\Omega} G(u) d x, 0<C_{2} \leq \frac{1}{2}$ is independent of $\gamma$.

Lemma 2.1.(Young's Inequality ${ }^{(\text {Lin,G.G.,2011) }}$ ) For any $\varepsilon>0$ and $a, b \geq 0$, then

$$
a b \leq \frac{\varepsilon^{p}}{p} a^{p}+\frac{1}{q \varepsilon^{q}},
$$

where $\frac{1}{p}+\frac{1}{q}=1, p>1, q>1$.

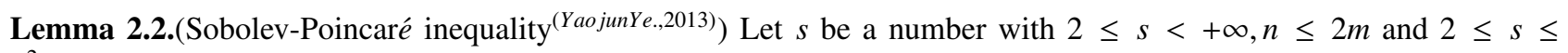
$\frac{2 m}{n-2 m}, n>2 m$. Then there is a constant $K$ depending on $\Omega$ and $s$ such that

$$
\|u\|_{s} \leq K\left\|(-\Delta)^{\frac{m}{2}} u\right\|, \forall u \in H_{0}^{m}(\Omega) .
$$

Lemma 2.3.(Gronwall's inequality ${ }^{(L i n, G . G ., 2011)}$ ) If $\forall t \in\left[t_{0},+\infty\right), y(t) \geq 0$ and $\frac{d y}{d t}+g y \leq h$, such that

$$
y(t) \leq y\left(t_{0}\right) e^{-g\left(t-t_{0}\right)}+\frac{h}{g}, t \geq t_{0},
$$

where $g>0, h \geq 0$ are constants.

Lemma 2.4. Assume $\left(H_{1}\right)-\left(H_{3}\right)$ hold, and $\left(u_{0}, u_{1}\right) \in H_{0}^{m}(\Omega) \times L^{2}(\Omega), f(x) \in L^{2}(\Omega)$. Then the solution $(u, v)$ of the problem $(1.1)-(1.3)$ satisfies $(u, v) \in L^{\infty}\left((0,+\infty) ; H_{0}^{m}(\Omega) \times L^{2}(\Omega)\right)$, and

$$
\left\|\nabla^{m} u\right\|^{2}+\|v\|^{2} \leq \frac{y(0)}{\min \left\{1, \frac{\beta^{q}-\varepsilon_{1}}{2}\right\}} e^{-\varepsilon_{1} C_{2} t}+\frac{\frac{\|f\|^{2}}{\varepsilon_{1}{ }^{2}}+C_{3}}{\varepsilon_{1} C_{2} \min \left\{1, \frac{\beta^{q}-\varepsilon_{1}}{2}\right\}} .
$$

where $v=u_{t}+\varepsilon_{1} u, 0<\varepsilon_{1}<\min \left\{\beta^{q}, \frac{\lambda_{1}{ }^{m}}{2 \lambda_{1}{ }^{m}+1}, \frac{\sqrt{\left(2+C_{2}\right)^{2}+16 \lambda_{1}{ }^{m}}-2-C_{2}}{4}\right\}, \lambda_{1}$ is the first eigenvalue of $-\Delta$ in $H_{0}^{1}(\Omega)$, and $y(0)=\left\|u_{1}+\varepsilon_{1} u_{0}\right\|^{2}+\frac{1}{\beta(q+1)}\left(\alpha+\beta\left\|\nabla^{m} u_{0}\right\|^{2}\right)^{q+1}-\varepsilon_{1}\left\|\nabla^{m} u_{0}\right\|^{2}+2 J\left(u_{0}\right)+2 C\left(\gamma_{1}\right)+\frac{q \beta^{q}}{q+1}$ 
,$C_{3}=\frac{(2 \alpha)^{q+1} \varepsilon_{1}}{q \beta}+m_{1}\left(2 C\left(\gamma_{1}\right)+\frac{q \beta^{q}}{q+1}\right)+\frac{4^{\frac{q+1}{q}} q}{2 \beta}+2 \varepsilon_{1} C\left(\gamma_{2}\right)$,

$m_{1}=\min \left\{2 \lambda_{1}{ }^{m}-2 \varepsilon_{1}-2 \varepsilon_{1}^{2}, \frac{\varepsilon_{1}(q+1)}{2}\right\}, \gamma_{1}=\frac{\beta^{q}-\varepsilon_{1}}{4}, \gamma_{2}=\frac{1}{2}-\varepsilon_{1}-\frac{\varepsilon_{1}}{2 \lambda_{1}{ }^{m}}$.

Thus, there exists $R_{0}$ and $t_{0}=t_{0}(\Omega)>0$, such that

$$
\|(u, v)\|_{H_{0}^{m} \times L^{2}}^{2}=\left\|\nabla^{m} u\right\|^{2}+\|v\|^{2} \leq R_{0}^{2}, \quad\left(t>t_{0}\right) .
$$

Proof. We take the scalar product in $L^{2}$ of equation (1.1) with $v=u_{t}+\varepsilon_{1} u$. Then

$$
\left(u_{t t}+(-\Delta)^{m} u_{t}+\left(\alpha+\beta\left\|\nabla^{m} u\right\|^{2}\right)^{q}(-\Delta)^{m} u+g(u), v\right)=(f(x), v) .
$$

By using Poincaré's inequality and Young's inequality, after a computation in (2.13), we have

$$
\begin{gathered}
\left(u_{t t}, v\right)=\frac{1}{2} \frac{d}{d t}\|v\|^{2}-\varepsilon_{1}\|v\|^{2}+\varepsilon_{1}^{2}(u, v) \\
\geq \frac{1}{2} \frac{d}{d t}\|v\|^{2}-\varepsilon_{1}\|v\|^{2}-\frac{\varepsilon_{1}^{2}}{2}\|u\|^{2}-\frac{\varepsilon_{1}^{2}}{2}\|v\|^{2} \\
\geq \frac{1}{2} \frac{d}{d t}\|v\|^{2}-\left(\varepsilon_{1}+\frac{\varepsilon_{1}^{2}}{2}\right)\|v\|^{2}-\frac{\varepsilon_{1}^{2}}{2 \lambda_{1}{ }^{m}}\left\|\nabla^{m} u\right\|^{2}, \\
\left((-\Delta)^{m} u_{t}, v\right)=-\frac{\varepsilon_{1}}{2} \frac{d}{d t}\left\|\nabla^{m} u\right\|^{2}+\left\|\nabla^{m} v\right\|^{2}-\varepsilon_{1}^{2}\left\|\nabla^{m} u\right\|^{2} \\
\geq-\frac{\varepsilon_{1}}{2} \frac{d}{d t}\left\|\nabla^{m} u\right\|^{2}+\lambda_{1}{ }^{m}\|v\|^{2}-\varepsilon_{1}^{2}\left\|\nabla^{m} u\right\|^{2}, \\
=\frac{1}{2}\left(\alpha+\beta\left\|\nabla^{m} u\right\|^{2}\right)^{q} \frac{d}{d t}\left\|\nabla^{m} u\right\|^{2}+\varepsilon_{1}\left(\alpha+\beta\left\|\nabla^{m} u\right\|^{2}\right)^{q}\left\|\nabla^{m} u\right\|^{2} \\
\frac{1}{2 \beta(q+1)} \frac{d}{d t}\left(\alpha+\beta\left\|\nabla^{m} u\right\|^{2}\right)^{q+1}+\frac{\varepsilon_{1}}{\beta}\left(\alpha+\beta\left\|\nabla^{m} u\right\|^{2}\right)^{q+1}-\frac{\alpha \varepsilon_{1}}{\beta}\left(\alpha+\beta\left\|\nabla^{m} u\right\|^{2}\right)^{q}, \\
(g(u), v) \quad=\frac{d}{d t} J(u)+\varepsilon_{1}(g(u), u), \\
(f(x), v) \leq \frac{1}{2 \varepsilon_{1}{ }^{2}}\|f\|^{2}+\frac{\varepsilon_{1}{ }^{2}}{2}\|v\|^{2} .
\end{gathered}
$$

Substituting (2.14)-(2.18) into (2.13), then

$$
\begin{aligned}
& \frac{d}{d t}\left[\|v\|^{2}+\frac{1}{\beta(q+1)}\left(\alpha+\beta\left\|\nabla^{m} u\right\|^{2}\right)^{q+1}-\varepsilon_{1}\left\|\nabla^{m} u\right\|^{2}+2 J(u)\right] \\
& +\left(2 \lambda_{1}{ }^{m}-2 \varepsilon_{1}-2 \varepsilon_{1}{ }^{2}\right)\|v\|^{2}+\frac{2 \varepsilon_{1}}{\beta}\left(\alpha+\beta\left\|\nabla^{m} u\right\|^{2}\right)^{q+1}-\frac{2 \alpha \varepsilon_{1}}{\beta}\left(\alpha+\beta\left\|\nabla^{m} u\right\|^{2}\right)^{q} \\
& -\left(\frac{\varepsilon_{1}{ }^{2}}{\lambda_{1}{ }^{m}}+2 \varepsilon_{1}{ }^{2}\right)\left\|\nabla^{m} u\right\|^{2}+2 \varepsilon_{1}(g(u), u) \leq \frac{\|f\|^{2}}{\varepsilon_{1}{ }^{2}} .
\end{aligned}
$$

Next, some of the items are estimated in (2.19). By Young's inequality, we have

$$
\begin{gathered}
\left\|\nabla^{m} u\right\|^{2} \leq \frac{1}{q+1}\left\|\nabla^{m} u\right\|^{2 q+2}+\frac{q}{q+1}, \\
\left\|\nabla^{m} u\right\|^{2} \leq \frac{\beta^{q}}{4(q+1)}\left\|\nabla^{m} u\right\|^{2 q+2}+\frac{q\left(\frac{4}{\beta^{q}}\right)^{\frac{1}{q}}}{q+1},
\end{gathered}
$$




$$
\left(\alpha+\beta\left\|\nabla^{m} u\right\|^{2}\right)^{q} \leq \frac{q}{2 \alpha(q+1)}\left(\alpha+\beta\left\|\nabla^{m} u\right\|^{2}\right)^{q+1}+\frac{(2 \alpha)^{q}}{q+1} .
$$

By (2.6), (2.20) and $\varepsilon_{1}<\beta^{q}$, we get

$$
\begin{aligned}
& \frac{1}{\beta(q+1)}\left(\alpha+\beta\left\|\nabla^{m} u\right\|^{2}\right)^{q+1}-\varepsilon_{1}\left\|\nabla^{m} u\right\|^{2}+2 J(u)+2 C\left(\gamma_{1}\right)+\frac{q \beta^{q}}{q+1} \\
\geq & \frac{\beta^{q}}{(q+1)}\left\|\nabla^{m} u\right\|^{2 q+2}-\varepsilon_{1}\left\|\nabla^{m} u\right\|^{2}+2 J(u)+2 C\left(\gamma_{1}\right)+\frac{q \beta^{q}}{q+1} \\
\geq & \left(\beta^{q}-\varepsilon_{1}\right)\left\|\nabla^{m} u\right\|^{2}+2 J(u)+2 C\left(\gamma_{1}\right) \\
\geq & 0,
\end{aligned}
$$

where $\gamma_{1}=\frac{\beta^{q}-\varepsilon_{1}}{4}$.

By (2.22), we have

$$
\begin{aligned}
& \frac{\varepsilon_{1}}{\beta}\left(\alpha+\beta\left\|\nabla^{m} u\right\|^{2}\right)^{q+1}-\frac{2 \alpha \varepsilon_{1}}{\beta}\left(\alpha+\beta\left\|\nabla^{m} u\right\|^{2}\right)^{q}+\frac{(2 \alpha)^{q+1} \varepsilon_{1}}{q \beta} \\
\geq & \frac{2 \alpha \varepsilon_{1}}{\beta q}\left(\alpha+\beta\left\|\nabla^{m} u\right\|^{2}\right)^{q} \\
\geq & 0 .
\end{aligned}
$$

Inserting (2.23)-(2.24) into (2.19), we obtain

$$
\begin{aligned}
& \frac{d}{d t}\left[\|v\|^{2}+\frac{1}{\beta(q+1)}\left(\alpha+\beta\left\|\nabla^{m} u\right\|^{2}\right)^{q+1}-\varepsilon_{1}\left\|\nabla^{m} u\right\|^{2}+2 J(u)+2 C\left(\gamma_{1}\right)+\frac{q \beta^{q}}{q+1}\right] \\
& +\left(2 \lambda_{1}{ }^{m}-2 \varepsilon_{1}-2 \varepsilon_{1}{ }^{2}\right)\|v\|^{2}+\frac{\varepsilon_{1}}{\beta}\left(\alpha+\beta\left\|\nabla^{m} u\right\|^{2}\right)^{q+1}-\left(\frac{\varepsilon_{1}{ }^{2}}{\lambda_{1}{ }^{m}}+2 \varepsilon_{1}{ }^{2}\right)\left\|\nabla^{m} u\right\|^{2} \\
& +2 \varepsilon_{1}(g(u), u) \leq \frac{\|f\|^{2}}{\varepsilon_{1}{ }^{2}}+\frac{(2 \alpha)^{q+1} \varepsilon_{1}}{q \beta} .
\end{aligned}
$$

In (2.25), by (2.7), (2.21) and $\varepsilon_{1}<\frac{\lambda_{1}{ }^{m}}{2 \lambda_{1}{ }^{m}+1}$, we have

$$
\begin{aligned}
& \left(2 \lambda_{1}{ }^{m}-2 \varepsilon_{1}-2 \varepsilon_{1}{ }^{2}\right)\|v\|^{2}+\frac{\varepsilon_{1}}{\beta}\left(\alpha+\beta\left\|\nabla^{m} u\right\|^{2}\right)^{q+1}-\left(\frac{\varepsilon_{1}{ }^{2}}{\lambda_{1}{ }^{m}}+2 \varepsilon_{1}{ }^{2}\right)\left\|\nabla^{m} u\right\|^{2}+2 \varepsilon_{1}(g(u), u) \\
\geq & \left(2 \lambda_{1}{ }^{m}-2 \varepsilon_{1}-2 \varepsilon_{1}{ }^{2}\right)\|v\|^{2}+\frac{\varepsilon_{1}}{2 \beta}\left(\alpha+\beta\left\|\nabla^{m} u\right\|^{2}\right)^{q+1} \\
& +\frac{\varepsilon_{1} \beta^{q}}{2}\left\|\nabla^{m} u\right\|^{2 q+2}-\left(\frac{\varepsilon_{1}{ }^{2}}{\lambda_{1}{ }^{m}}+2 \varepsilon_{1}{ }^{2}\right)\left\|\nabla^{m} u\right\|^{2}+2 \varepsilon_{1}(g(u), u) \\
\geq & \left(2 \lambda_{1}{ }^{m}-2 \varepsilon_{1}-2 \varepsilon_{1}{ }^{2}\right)\|v\|^{2}+\frac{\varepsilon_{1}}{2 \beta}\left(\alpha+\beta\left\|\nabla^{m} u\right\|^{2}\right)^{q+1} \\
& +\frac{\varepsilon_{1} \beta^{q}}{2}\left\|\nabla^{m} u\right\|^{2 q+2}-\varepsilon_{1}\left\|\nabla^{m} u\right\|^{2}+2 \varepsilon_{1} C_{2} J(u)-2 \varepsilon_{1} C\left(\gamma_{2}\right) \\
\geq & \left(2 \lambda_{1}{ }^{m}-2 \varepsilon_{1}-2 \varepsilon_{1}{ }^{2}\right)\|v\|^{2}+\frac{\varepsilon_{1}}{2 \beta}\left(\alpha+\beta\left\|\nabla^{m} u\right\|^{2}\right)^{q+1} \\
& +\left(2 q \varepsilon_{1}+\varepsilon_{1}\right)\left\|\nabla^{m} u\right\|^{2}+2 \varepsilon_{1} C_{2} J(u)-\frac{4^{\frac{q+1}{q}} q}{2 \beta}-2 \varepsilon_{1} C\left(\gamma_{2}\right) \\
\geq & m_{1}\left[\|v\|^{2}+\frac{1}{\beta(q+1)}\left(\alpha+\beta\left\|\nabla^{m} u\right\|^{2}\right)^{q+1}\right]+2 \varepsilon_{1} C_{2} J(u)-\frac{4^{\frac{q+1}{q}} q}{2 \beta}-2 \varepsilon_{1} C\left(\gamma_{2}\right) \\
\geq & m_{1}\left[\|v\|^{2}+\frac{1}{\beta(q+1)}\left(\alpha+\beta\left\|\nabla^{m} u\right\|^{2}\right)^{q+1}-\varepsilon_{1}\left\|\nabla^{m} u\right\|^{2}+2 C\left(\gamma_{1}\right)+\frac{q \beta^{q}}{q+1}\right] \\
& -m_{1}\left(2 C\left(\gamma_{1}\right)+\frac{q \beta^{q}}{q+1}\right)+2 \varepsilon_{1} C_{2} J(u)-\frac{4^{\frac{q+1}{q}} q}{2 \beta}-2 \varepsilon_{1} C\left(\gamma_{2}\right),
\end{aligned}
$$

where $\gamma_{2}=\frac{1}{2}-\varepsilon_{1}-\frac{\varepsilon_{1}}{2 \lambda_{1}{ }^{m}}$, and $m_{1}=\min \left\{2 \lambda_{1}{ }^{m}-2 \varepsilon_{1}-2 \varepsilon_{1}{ }^{2}, \frac{\varepsilon_{1}(q+1)}{2}\right\}$. 
Since $0<\varepsilon_{1}<\frac{\sqrt{\left(2+C_{2}\right)^{2}+16 \lambda_{1}{ }^{m}}-2-C_{2}}{4}$ and $0<C_{2} \leq \frac{1}{2}$, such that $m_{1} \geq \varepsilon_{1} C_{2}$.

Therefore, inserting (2.26) into (2.25), we have

$$
\begin{aligned}
& \frac{d}{d t}\left[\|v\|^{2}+\frac{1}{\beta(q+1)}\left(\alpha+\beta\left\|\nabla^{m} u\right\|^{2}\right)^{q+1}-\varepsilon_{1}\left\|\nabla^{m} u\right\|^{2}+2 J(u)+2 C\left(\gamma_{1}\right)+\frac{q \beta^{q}}{q+1}\right] \\
& +m_{1}\left[\|v\|^{2}+\frac{1}{\beta(q+1)}\left(\alpha+\beta\left\|\nabla^{m} u\right\|^{2}\right)^{q+1}-\varepsilon_{1}\left\|\nabla^{m} u\right\|^{2}+2 C\left(\gamma_{1}\right)+\frac{q \beta^{q}}{q+1}\right]+2 \varepsilon_{1} C_{2} J(u) \\
\leq & \frac{d}{d t}\left[\|v\|^{2}+\frac{1}{\beta(q+1)}\left(\alpha+\beta\left\|\nabla^{m} u\right\|^{2}\right)^{q+1}-\varepsilon_{1}\left\|\nabla^{m} u\right\|^{2}+2 J(u)+2 C\left(\gamma_{1}\right)+\frac{q \beta^{q}}{q+1}\right] \\
& +\varepsilon_{1} C_{2}\left[\|v\|^{2}+\frac{1}{\beta(q+1)}\left(\alpha+\beta\left\|\nabla^{m} u\right\|^{2}\right)^{q+1}-\varepsilon_{1}\left\|\nabla^{m} u\right\|^{2}+2 J(u)+2 C\left(\gamma_{1}\right)+\frac{q \beta^{q}}{q+1}\right] \\
\leq & \frac{\|f\|^{2}}{\varepsilon_{1}{ }^{2}}+C_{3},
\end{aligned}
$$

with $C_{3} \equiv \frac{(2 \alpha)^{q+1} \varepsilon_{1}}{q \beta}+m_{1}\left(2 C\left(\gamma_{1}\right)+\frac{q \beta^{q}}{q+1}\right)+\frac{4^{\frac{q+1}{q}} q}{2 \beta}+2 \varepsilon_{1} C\left(\gamma_{2}\right)$.

We set $y(t)=\|v\|^{2}+\frac{1}{\beta(q+1)}\left(\alpha+\beta\left\|\nabla^{m} u\right\|^{2}\right)^{q+1}-\varepsilon_{1}\left\|\nabla^{m} u\right\|^{2}+2 J(u)+2 C\left(\gamma_{1}\right)+\frac{q \beta^{q}}{q+1}$. Then, (2.27) is simplified as

$$
\frac{d}{d t} y(t)+\varepsilon_{1} C_{2} y(t) \leq \frac{\|f\|^{2}}{\varepsilon_{1}^{2}}+C_{3}
$$

From conclusion (2.23), we know $y(t) \geq 0$. So, by Gronwall's inequality and (2.23), we obtain

$$
\|v\|^{2}+\frac{\beta^{q}-\varepsilon_{1}}{2}\left\|\nabla^{m} u\right\|^{2} \leq y(t) \leq y(0) e^{-\varepsilon_{1} C_{2} t}+\frac{\frac{\|f\|^{2}}{\varepsilon_{1}^{2}}+C_{3}}{\varepsilon_{1} C_{2}},
$$

where $y(0)=\left\|u_{1}+\varepsilon_{1} u_{0}\right\|^{2}+\frac{1}{\beta(q+1)}\left(\alpha+\beta\left\|\nabla^{m} u_{0}\right\|^{2}\right)^{q+1}-\varepsilon_{1}\left\|\nabla^{m} u_{0}\right\|^{2}+2 J\left(u_{0}\right)+2 C\left(\gamma_{1}\right)+\frac{q \beta^{q}}{q+1}$.

Therefore, we get

$$
\|(u, v)\|_{H_{0}^{m} \times L^{2}}^{2}=\left\|\nabla^{m} u\right\|^{2}+\|v\|^{2} \leq \frac{y(0)}{\min \left\{1, \frac{\beta^{q}-\varepsilon_{1}}{2}\right\}} e^{-\varepsilon_{1} C_{2} t}+\frac{\frac{\|f\|^{2}}{\varepsilon_{1}{ }^{2}}+C_{3}}{\varepsilon_{1} C_{2} \min \left\{1, \frac{\beta^{q}-\varepsilon_{1}}{2}\right\}} .
$$

Then,

$$
\varlimsup_{t \rightarrow \infty}\|(u, v)\|_{H_{0}^{m} \times L^{2}}^{2}=\left\|\nabla^{m} u\right\|^{2}+\|v\|^{2} \leq \frac{\frac{\|f\|^{2}}{\varepsilon_{1}^{2}}+C_{3}}{\varepsilon_{1} C_{2} \min \left\{1, \frac{\beta^{q}-\varepsilon_{1}}{2}\right\}} .
$$

So, there exist $R_{0}$ and $t_{0}=t_{0}(\Omega)>0$, such that

$$
\|(u, v)\|_{H_{0}^{m} \times L^{2}}^{2}=\left\|\nabla^{m} u\right\|^{2}+\|v\|^{2} \leq R_{0}^{2}, \quad\left(t>t_{0}\right) .
$$

Lemma 2.5. In addition to the assumptions of Lemma 2.4., $\left(H_{1}\right)-\left(H_{4}\right)$ hold.If $\left(H_{5}\right): f(x) \in H_{0}^{m}(\Omega)$, and $\left(u_{0}, u_{1}\right) \in$ $H^{2 m}(\Omega) \cap H_{0}^{m}(\Omega) \times H_{0}^{m}(\Omega)$. Then the solution $(u, v)$ of the problems $(1.1)-(1.3)$ satisfies $(u, v) \in L^{\infty}\left((0,+\infty) ; H^{2 m}(\Omega) \cap H_{0}^{m}(\Omega) \times H_{0}^{m}(\Omega)\right)$, and

$$
\left\|\nabla^{m} v\right\|^{2}+\left\|\Delta^{m} u\right\|^{2} \leq \frac{z(0)}{\min \left\{1, \mu-\varepsilon_{2}\right\}} e^{-m_{2} t}+\frac{C_{5}+\frac{1}{\varepsilon_{2}^{2}}\left\|\nabla^{m} f\right\|^{2}}{\min \left\{1, \mu-\varepsilon_{2}\right\} m_{2}},
$$

where $(-\Delta)^{m} v=(-\Delta)^{m} u_{t}+\varepsilon_{2}(-\Delta)^{m} u, \lambda_{1}$ is the first eigenvalue of $-\Delta$ in $H_{0}^{1}(\Omega)$, and $z(0)=\left\|\nabla^{m} u_{1}+\varepsilon \nabla^{m} u_{0}\right\|^{2}+$ $\left(\mu-\varepsilon_{2}\right)\left\|\Delta^{m} u_{0}\right\|^{2}, m_{2}=\min \left\{\lambda_{1}{ }^{m}-2 \varepsilon_{2}-2 \varepsilon_{2}{ }^{2}, \frac{-\frac{\varepsilon_{2}{ }^{2}}{\lambda_{1} m^{m}}-2 \varepsilon_{2}{ }^{2}+2 \alpha^{q} \varepsilon_{2}}{\mu-\varepsilon_{2}}\right\}$.

Thus, there exists $R_{1}$ and $t_{1}=t_{1}(\Omega)>0$, such that

$$
\|(u, v)\|_{H^{2 m} \cap H_{0}^{m} \times H_{0}^{m}}=\left\|\Delta^{m} u\right\|^{2}+\left\|\nabla^{m} v\right\|^{2} \leq R_{1}^{2}, \quad\left(t>t_{1}\right) .
$$


Proof. Taking $L^{2}$-inner product by $(-\Delta)^{m} v=(-\Delta)^{m} u_{t}+\varepsilon_{2}(-\Delta)^{m} u$ in (1.1), we have

$$
\left(u_{t t}+(-\Delta)^{m} u_{t}+\left(\alpha+\beta\left\|\nabla^{m} u\right\|^{2}\right)^{q}(-\Delta)^{m} u+g(u),(-\Delta)^{m} v\right)=\left(f(x),(-\Delta)^{m} v\right) .
$$

After a computation in (2.35) one by one, as follow

$$
\begin{aligned}
&\left(u_{t t},(-\Delta)^{m} v\right)=\frac{1}{2} \frac{d}{d t}\left\|\nabla^{m} v\right\|^{2}-\varepsilon_{2}\left\|\nabla^{m} v\right\|^{2}+\varepsilon_{2}^{2}\left(\nabla^{m} u, \nabla^{m} v\right) \\
& \geq \frac{1}{2} \frac{d}{d t}\left\|\nabla^{m} v\right\|^{2}-\varepsilon_{2}\left\|\nabla^{m} v\right\|^{2}-\frac{\varepsilon_{2}^{2}}{2 \lambda_{1}{ }^{m}}\left\|\Delta^{m} u\right\|^{2}-\frac{\varepsilon_{2}^{2}}{2}\left\|\nabla^{m} v\right\|^{2} . \\
&\left((-\Delta)^{m} u_{t},(-\Delta)^{m} v\right)=\left\|\Delta^{m} v\right\|^{2}-\frac{\varepsilon_{2}}{2} \frac{d}{d t}\left\|\Delta^{m} u\right\|^{2}-\varepsilon_{2}^{2}\left\|\Delta^{m} u\right\|^{2} .
\end{aligned}
$$

By Lemma 2.4., we know it exists $C_{4}$, such that

$$
\alpha^{q} \leq\left(\alpha+\beta\left\|\nabla^{m} u\right\|^{2}\right)^{q} \leq C_{4} .
$$

We set

$$
\mu= \begin{cases}\alpha^{q}, & \frac{d}{d t}\left\|\Delta^{m} u\right\|^{2} \geq 0 \\ C_{4}, & \frac{d}{d t}\left\|\Delta^{m} u\right\|^{2} \leq 0 .\end{cases}
$$

By (2.38) and (2.39), we get

$$
\begin{aligned}
& \left(\left(\alpha+\beta\left\|\nabla^{m} u\right\|^{2}\right)^{q}(-\Delta)^{m} u,(-\Delta)^{m} v\right) \\
= & \frac{\left(\alpha+\beta\left\|\nabla^{m} u\right\|^{2}\right)^{q}}{2} \frac{d}{d t}\left\|\Delta^{m} u\right\|^{2}+\varepsilon_{2}\left(\alpha+\beta\left\|\nabla^{m} u\right\|^{2}\right)^{q}\left\|\Delta^{m} u\right\|^{2} \\
\geq & \frac{\mu}{2} \frac{d}{d t}\left\|\Delta^{m} u\right\|^{2}+\varepsilon_{2} \alpha^{q}\left\|\Delta^{m} u\right\|^{2} .
\end{aligned}
$$

By Young's inequality, we get

$$
\left(g(u),(-\Delta)^{m} v\right) \geq-\|g(u)\|\left\|\Delta^{m} v\right\| \geq-\frac{\|g(u)\|^{2}}{2}-\frac{\left\|\Delta^{m} v\right\|^{2}}{2} .
$$

Next to estimate $\|g(u)\|^{2}$ in (2.41). By $\left(H_{4}\right):|g(s)| \leq C_{1}\left(1+|s|^{p}\right)$ and Young's inequality, we have

$$
\begin{aligned}
\|g(u)\|^{2} & \leq \int_{\Omega} C_{1}^{2}\left(1+|u|^{p}\right)^{2} d x \\
& \leq \int_{\Omega}\left(C_{1}^{2}+2 C_{1}^{2}|u|^{p}+C_{1}^{2}|u|^{2 p}\right) d x \\
& \leq \int_{\Omega}\left(2 C_{1}^{2}+2 C_{1}^{2}|u|^{2 p}\right) d x \\
& \leq 2 C_{1}^{2}|\Omega|+2 C_{1}^{2}\|u\|_{L^{2 p}(\Omega)}^{2 p} .
\end{aligned}
$$

By $1 \leq p \leq \frac{n+2 m}{n-2 m}, n>2 m(1 \leq p<+\infty, n \leq 2 m)$. So, there exists $K>0$, such that $\|u\|_{L^{2 p}(\Omega)} \leq K\left\|\nabla^{m} u\right\| \cdot\left\|\nabla^{m} u\right\|$ bounded by Lemma 2.4. Then, (2.42) turns into

$$
\|g(u)\|^{2} \leq C_{5}\left(p, C_{1}, K,|\Omega|\right) .
$$

Collecting with (2.43), from (2.41) we have

$$
\left(g(u),(-\Delta)^{m} v\right) \geq-\frac{C_{5}}{2}-\frac{\left\|\Delta^{m} v\right\|^{2}}{2} .
$$

By $f(x) \in H_{0}^{m}(\Omega)$ and Young's inequality, we obtain

$$
\left(f(x),(-\Delta)^{m} v\right)=\left(\nabla^{m} f(x), \nabla^{m} v\right) \leq \frac{1}{2 \varepsilon_{2}^{2}}\left\|\nabla^{m} f\right\|^{2}+\frac{\varepsilon_{2}^{2}}{2}\left\|\nabla^{m} v\right\|^{2} .
$$


Integrating (2.36)-(2.40),(2.44)-(2.45), from (2.35) entails

$$
\begin{aligned}
& \frac{d}{d t}\left[\left\|\nabla^{m} v\right\|^{2}+\left(\mu-\varepsilon_{2}\right)\left\|\Delta^{m} u\right\|^{2}\right]+\left\|\Delta^{m} v\right\|^{2} \\
- & 2\left(\varepsilon_{2}+\varepsilon_{2}{ }^{2}\right)\left\|\nabla^{m} v\right\|^{2}+\left(-\frac{\varepsilon_{2}{ }^{2}}{\lambda_{1}{ }^{m}}-2 \varepsilon_{2}{ }^{2}+2 \alpha^{q} \varepsilon_{2}\right)\left\|\Delta^{m} u\right\|^{2} \\
\leq & C_{5}+\frac{1}{\varepsilon_{2}{ }^{2}}\left\|\nabla^{m} f\right\|^{2}
\end{aligned}
$$

By Poincaré inequality, such that $\lambda_{1}^{m}\left\|\nabla^{m} v\right\|^{2} \leq\left\|\Delta^{m} v\right\|^{2}$. So, (2.46) turns into

$$
\begin{aligned}
& \frac{d}{d t}\left[\left\|\nabla^{m} v\right\|^{2}+\left(\mu-\varepsilon_{2}\right)\left\|\Delta^{m} u\right\|^{2}\right]+\left(\lambda_{1}{ }^{m}-2 \varepsilon_{2}-2 \varepsilon_{2}{ }^{2}\right)\left\|\nabla^{m} v\right\|^{2} \\
& +\left(-\frac{\varepsilon_{2}{ }^{2}}{\lambda_{1}{ }^{m}}-2 \varepsilon_{2}{ }^{2}+2 \alpha^{q} \varepsilon_{2}\right)\left\|\Delta^{m} u\right\|^{2} \leq C_{5}+\frac{1}{\varepsilon_{2}{ }^{2}}\left\|\nabla^{m} f\right\|^{2} .
\end{aligned}
$$

Taking $m_{2}=\min \left\{\lambda_{1}{ }^{m}-2 \varepsilon_{2}-2 \varepsilon_{2}{ }^{2}, \frac{-\frac{\varepsilon_{2}{ }^{m}}{\lambda_{1}{ }^{m}}-2 \varepsilon_{2}{ }^{2}+2 \alpha^{q} \varepsilon_{2}}{\mu-\varepsilon_{2}}\right\}$, then

$$
\frac{d}{d t} z(t)+m_{2} z(t) \leq \frac{1}{\varepsilon_{2}^{2}}\left\|\nabla^{m} f\right\|^{2}+C_{5}
$$

where $z(t)=\left\|\nabla^{m} v\right\|^{2}+\left(\mu-\varepsilon_{2}\right)\left\|\Delta^{m} u\right\|^{2}$.

By Gronwall's inequality, we have

$$
z(t) \leq z(0) e^{-m_{2} t}+\frac{\frac{1}{\varepsilon^{2}}\left\|\nabla^{m} f\right\|^{2}+C_{5}}{m_{2}},
$$

where $z(0)=\left\|\nabla^{m} u_{1}+\varepsilon_{2} \nabla^{m} u_{0}\right\|^{2}+\left(\mu-\varepsilon_{2}\right)\left\|\Delta^{m} u_{0}\right\|^{2}$.

Therefore, we have

$$
\left\|\nabla^{m} v\right\|^{2}+\left\|\Delta^{m} u\right\|^{2} \leq \frac{z(0)}{\min \left\{1, \mu-\varepsilon_{2}\right\}} e^{-m_{2} t}+\frac{C_{5}+\frac{1}{\varepsilon_{2}}\left\|\nabla^{m} f\right\|^{2}}{\min \left\{1, \mu-\varepsilon_{2}\right\} m_{2}} .
$$

Then

$$
\varlimsup_{t \rightarrow \infty}\|(u, v)\|_{H^{2 m}}^{2} \cap H_{0}^{m} \times H_{0}^{m}=\left\|\Delta^{m} u\right\|^{2}+\left\|\nabla^{m} v\right\|^{2} \leq \frac{C_{5}+\frac{1}{\varepsilon_{2}^{2}}\left\|\nabla^{m} f\right\|^{2}}{\min \left\{1, \mu-\varepsilon_{2}\right\} m_{2}} .
$$

So,there exists $R_{1}$ and $t_{1}=t_{1}(\Omega)>0$, such that

$$
\|(u, v)\|_{H^{2 m} \cap H_{0}^{m} \times H_{0}^{m}}=\left\|\Delta^{m} u\right\|^{2}+\left\|\nabla^{m} v\right\|^{2} \leq R_{1}^{2}, \quad\left(t>t_{1}\right) .
$$

\section{Global Attractor}

\subsection{The Existence and Uniqueness of Solution}

Theorem 3.1. Assume $\left(H_{1}\right)-\left(H_{4}\right)$ hold, and $\left(u_{0}, u_{1}\right) \in H^{2 m}(\Omega) \cap H_{0}^{m}(\Omega) \times H_{0}^{m}(\Omega), f(x) \in H_{0}^{m}(\Omega), v=u_{t}+\varepsilon_{1} u$. So Equation (1.1) exists a unique smooth solution

$$
(u(x, t), v(x, t)) \in L^{\infty}\left((0,+\infty) ; H^{2 m}(\Omega) \bigcap H_{0}^{m}(\Omega) \times H_{0}^{m}(\Omega)\right) .
$$

Proof. By the Galerkin method, Lemma 2.4. and Lemma 2.5. , we can easily obtain the existence of Solutions. Next, we prove the uniqueness of Solutions in detail.

Assume $u, v$ are two solutions of the problems (1.1)-(1.3), let $w=u-v$, then $w(x, 0)=w_{0}(x)=0, w_{t}(x, 0)=w_{1}(x)=0$ and the two equations subtract and obtain

$$
w_{t t}+(-\Delta)^{m} w_{t}+\left(\alpha+\beta\left\|\nabla^{m} u\right\|^{2}\right)^{q}(-\Delta)^{m} u-\left(\alpha+\beta\left\|\nabla^{m} v\right\|^{2}\right)^{q}(-\Delta)^{m} v+g(u)-g(v)=0 .
$$


By multiplying (3.2) by $w_{t}$, we get

$$
\begin{aligned}
&\left(w_{t t}+\right.\left.(-\Delta)^{m} w_{t}+\left(\alpha+\beta\left\|\nabla^{m} u\right\|^{2}\right)^{q}(-\Delta)^{m} u-\left(\alpha+\beta\left\|\nabla^{m} v\right\|^{2}\right)^{q}(-\Delta)^{m} v+g(u)-g(v), w_{t}\right)=0 . \\
&\left(w_{t t}, w_{t}\right)=\frac{1}{2} \frac{d}{d t}\left\|w_{t}\right\|^{2}, \\
&\left((-\Delta)^{m} w_{t}, w_{t}\right)=\left\|\nabla^{m} w_{t}\right\|^{2}, \\
&\left(\left(\alpha+\beta\left\|\nabla^{m} u\right\|^{2}\right)^{q}(-\Delta)^{m} u-\left(\alpha+\beta\left\|\nabla^{m} v\right\|^{2}\right)^{q}(-\Delta)^{m} v, w_{t}\right) \\
&=\left(\alpha+\beta\left\|\nabla^{m} u\right\|^{2}\right)^{q}\left((-\Delta)^{m} w, w_{t}\right)+\left[\left(\alpha+\beta\left\|\nabla^{m} u\right\|^{2}\right)^{q}-\left(\alpha+\beta\left\|\nabla^{m} v\right\|^{2}\right)^{q}\right]\left((-\Delta)^{m} v, w_{t}\right) \\
&=\frac{1}{2} \frac{d}{d t}\left[\left\|\nabla^{m} w\right\|^{2}\left(\alpha+\beta\left\|\nabla^{m} u\right\|^{2}\right)^{q}\right]-\frac{1}{2}\left\|\nabla^{m} w\right\|^{2} \frac{d}{d t}\left(\alpha+\beta\left\|\nabla^{m} u\right\|^{2}\right)^{q} \\
&+\left[\left(\alpha+\beta\left\|\nabla^{m} u\right\|^{2}\right)^{q}-\left(\alpha+\beta\left\|\nabla^{m} v\right\|^{2}\right)^{q}\right]\left((-\Delta)^{m} v, w_{t}\right) \\
&=\frac{1}{2} \frac{d}{d t}\left[\left\|\nabla^{m} w\right\|^{2}\left(\alpha+\beta\left\|\nabla^{m} u\right\|^{2}\right)^{q}\right]-q \beta\left(\alpha+\beta\left\|\nabla^{m} u\right\|^{2}\right)^{q-1}\left\|\nabla^{m} u\right\|\left\|\nabla^{m} u_{t}\right\|\left\|\nabla^{m} w\right\|^{2} \\
&+\left[\left(\alpha+\beta\left\|\nabla^{m} u\right\|^{2}\right)^{q}-\left(\alpha+\beta\left\|\nabla^{m} v\right\|^{2}\right)^{q}\right]\left((-\Delta)^{m} v, w_{t}\right) .
\end{aligned}
$$

Exploiting (3.4)-(3.6), we receive

$$
\begin{aligned}
& \frac{d}{d t}\left[\left\|w_{t}\right\|^{2}+\left(\alpha+\beta\left\|\nabla^{m} u\right\|^{2}\right)^{q}\left\|\nabla^{m} w\right\|^{2}\right]+2\left\|\nabla^{m} w_{t}\right\|^{2} \\
= & 2 q \beta\left(\alpha+\beta\left\|\nabla^{m} u\right\|^{2}\right)^{q-1}\left\|\nabla^{m} u\right\|\left\|\nabla^{m} u_{t}\right\|\left\|\nabla^{m} w\right\|^{2} \\
& -2\left[\left(\alpha+\beta\left\|\nabla^{m} u\right\|^{2}\right)^{q}-\left(\alpha+\beta\left\|\nabla^{m} v\right\|^{2}\right)^{q}\right]\left((-\Delta)^{m} v, w_{t}\right)-2(g(u)-g(v))=0 .
\end{aligned}
$$

In (3.7), according to Lemma 2.4. and Lemma 2.5., such that

$$
\begin{aligned}
& 2 q \beta\left(\alpha+\beta\left\|\nabla^{m} u\right\|^{2}\right)^{q-1}\left\|\nabla^{m} u\right\|\left\|\nabla^{m} u_{t}\right\|\left\|\nabla^{m} w\right\|^{2} \\
& -2\left[\left(\alpha+\beta\left\|\nabla^{m} u\right\|^{2}\right)^{q}-\left(\alpha+\beta\left\|\nabla^{m} v\right\|^{2}\right)^{q}\right]\left((-\Delta)^{m} v, w_{t}\right) \\
\leq & C_{6}\left\|\nabla^{m} w\right\|^{2}+4 q \beta \xi\left(\alpha+\beta \xi^{2}\right)^{q-1}\left\|\Delta^{m} v\right\|\left\|w_{t}\right\|\left\|\nabla^{m} w\right\| \\
\leq & C_{6}\left\|\nabla^{m} w\right\|^{2}+C_{7}\left\|w_{t}\right\|\left\|\nabla^{m} w\right\| \\
\leq & \left(C_{6}+\frac{C_{7}}{2}\right)\left(\left\|w_{t}\right\|^{2}+\left\|\nabla^{m} w\right\|^{2}\right) .
\end{aligned}
$$

where $\min _{t \in[0,+\infty)}\left\{\left\|\nabla^{m} u\right\|,\left\|\nabla^{m} v\right\|\right\}<\xi<\max _{t \in[0,+\infty)}\left\{\left\|\nabla^{m} u\right\|,\left\|\nabla^{m} v\right\|\right\}, C_{6}>0$, and $C_{7}>0$ are constants.

By $\left(H_{4}\right)$, Lemma 2.2., Lemma 2.4. and Lemma 2.5., we obtain

$$
\begin{aligned}
& \left|-2\left(g(u)-g(v), w_{t}\right)\right| \\
\leq & 2\|g(u)-g(v)\|\left\|w_{t}\right\| \\
\leq & 2\left\|\int_{0}^{1} \frac{d}{d s} g(s u+(1-s) v) d s\right\|\left\|w_{t}\right\| \\
\leq & C_{1}\left\|\int_{0}^{1}\left[1+|s u+(1-s) v|^{p-1}\right] d s \times w\right\|\left\|w_{t}\right\| \\
\leq & C_{1}\left\|\left[1+(|u|+|v|)^{p-1}\right]|w|\right\|\left\|w_{t}\right\| \\
\leq & C_{1}\left[1+\left(\left\|\Delta^{m} u\right\|^{p+1}+\left\|\Delta^{m} v\right\|^{p+1}\right)\right]\left\|w_{t}\right\|\|w\| \\
\leq & C_{8}\left(\left\|w_{t}\right\|^{2}+\left\|\nabla^{m} w\right\|^{2}\right)
\end{aligned}
$$


where $C_{8}>0$ is constant. From the above, we have

$$
\begin{aligned}
& \frac{d}{d t}\left[\left\|w_{t}\right\|^{2}+\left(\alpha+\beta\left\|\nabla^{m} u\right\|^{2}\right)^{q}\left\|\nabla^{m} w\right\|^{2}\right] \\
\leq & \left(C_{6}+\frac{C_{7}}{2}+C_{8}\right)\left(\left\|w_{t}\right\|^{2}+\left\|\nabla^{m} w\right\|^{2}\right) \\
\leq & \left(C_{6}+\frac{C_{7}}{2}+C_{8}\right)\left\|w_{t}\right\|^{2}+\frac{C_{6}+\frac{C_{7}}{2}+C_{8}}{\left(\alpha+\beta\left\|\nabla^{m} u\right\|^{2}\right)^{q}}\left(\alpha+\beta\left\|\nabla^{m} u\right\|^{2}\right)^{q}\left\|\nabla^{m} w\right\|^{2} \\
\leq & \max \left\{C_{6}+\frac{C_{7}}{2}+C_{8}, \frac{C_{6}+\frac{C_{7}}{2}+C_{8}}{\alpha^{2 q}}\right\}\left[\left\|w_{t}\right\|^{2}+\left(\alpha+\beta\left\|\nabla^{m} u\right\|^{2}\right)^{q}\left\|\nabla^{m} w\right\|^{2}\right] .
\end{aligned}
$$

By using Gronwall's inequality for (3.10), we obtain

$$
\begin{aligned}
& \left\|w_{t}\right\|^{2}+\left(\alpha+\beta\left\|\nabla^{m} u\right\|^{2}\right)^{q}\left\|\nabla^{m} w\right\|^{2} \\
\leq & {\left[\left\|w_{t}(0)\right\|^{2}+\left(\alpha+\beta\left\|\nabla^{m} u_{0}\right\|^{2}\right)^{q}\left\|\nabla^{m} w(0)\right\|^{2}\right] e^{C_{9} t}=0, }
\end{aligned}
$$

where $C_{9}=\max \left\{C_{6}+\frac{C_{7}}{2}+C_{8}, \frac{C_{6}+\frac{C_{7}}{2}+C_{8}}{\alpha^{2 q}}\right\}>0$.

Hence, we can get $\left\|w_{t}\right\|^{2}+\left(\alpha+\beta\left\|\nabla^{m} u\right\|^{2}\right)^{q}\left\|\nabla^{m} w\right\|^{2}=0$. That shows that

$$
\left\|w_{t}\right\|^{2}=0, \quad\left(\alpha+\beta\left\|\nabla^{m} u\right\|^{2}\right)^{q}\left\|\nabla^{m} w\right\|^{2} .
$$

That is

$$
w(x, t)=0 .
$$

Therefore,

$$
u=v
$$

So, we get the uniqueness of the solution.

\subsection{The Existence of Global Attractor}

Theorem 3.2. ${ }^{(L i n, G . G ., 2011)}$ Let $E$ be a Banach space, and $\{S(t)\}(t \geq 0)$ are the semigroup operator on $E_{0} . S(t): E_{0} \rightarrow$ $E_{0}, S(t+\tau)=S(t) S(\tau)(\forall t, \tau \geq 0), S(0)=I$, where I is a unit operator.Set $S(t)$ satisfy the follow conditions:

1) $S(t)$ is uniformly bounded, namely $\forall R>0,\|u\|_{E} \leq R$, it exists a constant $C(R)$, so that

$$
\|S(t) u\|_{E} \leq C(R) \quad(t \in[0,+\infty)) ;
$$

2) It exists a bounded absorbing set $B_{0} \subset E$, namely, $\forall B \subset E$, it exists a constant $t_{0}$, so that

$$
S(t) B \subset B_{0} \quad\left(t \geq t_{0}\right) ;
$$

where $B_{0}$ and $B$ are bounded sets.

3) When $t>0, S(t)$ is a completely continuous operator. Therefore, the semigroup operator $\mathrm{S}(\mathrm{t})$ exists a compact global attractor $\mathcal{A}$.

Theorem 3.3. Under the assume of Lemma 2.4., Lemma 2.5. and Theorem 3.1., equations have global attractor

$$
\mathcal{A}=\omega\left(B_{0}\right)=\bigcap_{\tau \geq 0} S(t) B_{0},
$$

where $B_{0}=\left\{(u, v) \in H^{2 m}(\Omega) \cap H_{0}^{m}(\Omega) \times H_{0}^{m}(\Omega):\|(u, v)\|_{H^{2 m} \cap H_{0}^{m} \times H_{0}^{m}}=\|u\|_{H^{2 m} \cap H_{0}^{m}}+\|v\|_{H_{0}^{m}} \leq R_{0}+R_{1}\right\}, B_{0}$ is the bounded absorbing set of $H^{2 m} \times H_{0}^{m}$ and satisfies

1) $S(t) \mathcal{A}=\mathcal{A}, t>0$;

2) $\lim _{t \rightarrow \infty} \operatorname{dist}(S(t) B, \mathcal{A})=0$, here $B \subset H^{2 m} \cap H_{0}^{m} \times H_{0}^{m}$ and it is a bounded set, 


$$
\operatorname{dist}(S(t) B, \mathcal{A})=\sup _{x \in B}\left(\inf _{y \in \mathcal{A}}\|S(t) x-y\|_{H^{2 m} \cap H_{0}^{m} \times H_{0}^{m}}\right) \longrightarrow 0, t \longrightarrow \infty .
$$

Proof. Under the conditions of Theorem 3.1., it exists the solution semigroup $\mathrm{S}(\mathrm{t}), S(t): H^{2 m} \cap H_{0}^{m} \times H_{0}^{m} \rightarrow H^{2 m} \cap H_{0}^{m} \times$ $H_{0}^{m}$, here $E_{1}=H^{2 m}(\Omega) \cap H_{0}^{m}(\Omega) \times H_{0}^{m}(\Omega)$.

(1) From Lemma 2.4. to Lemma 2.5., we can get that $\forall B \subset H^{2 m}(\Omega) \cap H_{0}^{m}(\Omega) \times H_{0}^{m}(\Omega)$ is a bounded set that includes in the ball $\left\{\|(u, v)\|_{H^{2 m} \cap H_{0}^{m} \times H_{0}^{m}} \leq R\right\}$,

$$
\begin{aligned}
\left\|S(t)\left(u_{0}, v_{0}\right)\right\|_{H^{2 m} \cap H_{0}^{m} \times H_{0}^{m}}^{2} & =\|u\|_{H^{2 m} \cap H_{0}^{m}}^{2}+\|v\|_{H_{0}^{m}}^{2} \\
& \leq\left\|u_{0}\right\|_{H^{2 m} \cap H_{0}^{m}}^{2}+\left\|v_{0}\right\|_{H_{0}^{m}}^{2}+C \\
& \leq R_{1}^{2}+C,\left(t \geq 0,\left(u_{0}, v_{0}\right) \in B\right) .
\end{aligned}
$$

This shows that $S(t)(t \geq 0)$ is uniformly bounded in $H^{2 m}(\Omega) \cap H_{0}^{m}(\Omega) \times H_{0}^{m}(\Omega)$.

(2) Furthermore, for any $\left(u_{0}, v_{0}\right) \in H^{2 m}(\Omega) \cap H_{0}^{m}(\Omega) \times H_{0}^{m}(\Omega)$, when $t \geq \max \left\{t_{0}, t_{1}\right\}$, we have

$$
\left\|S(t)\left(u_{0}, v_{0}\right)\right\|_{H^{2 m} \cap H_{0}^{m} \times H_{0}^{m}}^{2}=\|u\|_{H^{2 m} \cap H_{0}^{m}}^{2}+\|v\|_{H_{0}^{m}}^{2} \leq R_{0}^{2}+R_{1}^{2} .
$$

So we get $B_{0}$ is the bounded absorbing set.

(3) Since $E_{1}:=H^{2 m}(\Omega) \cap H_{0}^{m}(\Omega) \times H_{0}^{m}(\Omega) \hookrightarrow E_{0}:=H_{0}^{m}(\Omega) \times L^{2}(\Omega)$ is compact embedded, which means that the bounded set in $E_{1}$ is the compact set in $E_{0}$, so the semigroup operator $\mathrm{S}(\mathrm{t})$ exists a compact global attractor $\mathcal{A}$.

\section{The Estimates of the Upper Bounds of Hausdorff Dimensions for the Global Attractor}

\subsection{Differentiability of the Semigroup}

In order to estimate dimensions, we suppose: $\left(H_{5}\right)$ for every $M>0$, there exist $k=k(M)$, such that:

$$
\left\|g^{\prime}\left(u_{1}\right)-g^{\prime}\left(u_{2}\right)\right\|_{L\left(H_{0}^{m}(\Omega), L^{2}(\Omega)\right)} \leq k\left\|\nabla^{m} u_{1}-\nabla^{m} u_{2}\right\|^{\delta_{1}},
$$

for any $u_{1}, u_{2} \in H_{0}^{m}(\Omega),\left\|\nabla^{m} u_{1}\right\| \leq M,\left\|\nabla^{m} u_{2}\right\| \leq M, \delta_{1}>0$.

We define $A=-\Delta, E_{0}=H_{0}^{m}(\Omega) \times L^{2}(\Omega)$. The inner product and the norm in $E_{0}$ space are defined as follows:

$\forall \varphi_{i}=\left(u_{i}, v_{i}\right) \in E_{0},(i=1,2)$, we have

$$
\begin{gathered}
\left(\varphi_{1}, \varphi_{2}\right)_{E_{0}}=\left(A^{\frac{m}{2}} u_{1}, A^{\frac{m}{2}} u_{2}\right)+\left(v_{1}, v_{2}\right), \\
\left\|\varphi_{1}\right\|_{E_{0}}^{2}=\left(\varphi_{1}, \varphi_{1}\right)_{E_{0}}=\left\|A^{\frac{m}{2}} u_{1}\right\|^{2}+\left\|v_{1}\right\|^{2} .
\end{gathered}
$$

Setting $\forall \varphi=(u, v)^{T} \in E_{0}, v=u_{t}+\varepsilon u, 0<\varepsilon<\min \left\{1, \frac{\lambda_{1}^{m}}{2}, \frac{-\frac{5}{2}-\lambda_{1}^{m}+\sqrt{\left(\frac{5}{2}+\lambda_{1}^{m}\right)^{2}+4 \lambda_{1}{ }^{m}}}{2}\right\}$, the equation (1.1) is equivalent to

$$
\varphi_{t}+H(\varphi)=F(\varphi)
$$

where

$$
\begin{gathered}
H(\varphi)=\left[\begin{array}{c}
\varepsilon u-v \\
\left.-\varepsilon v+A^{m} v+\varepsilon^{2} u+(1-\varepsilon) A^{m} u\right]
\end{array}\right], \\
F(\varphi)=\left[\begin{array}{c}
0 \\
{\left[1-\left(\alpha+\beta\left\|\nabla^{m} u\right\|^{2}\right)^{q}\right] A^{m} u-g(u)+f(x)}
\end{array}\right] .
\end{gathered}
$$

Lemma 4.1.1. For any $\varphi=(u, v)^{T} \in E_{0}$, we have

$$
(H(\varphi), \varphi)_{E_{0}} \geq \frac{\varepsilon}{4}\|\varphi\|_{E_{0}}^{2}+\frac{1}{2}\left\|A^{\frac{m}{2}} v\right\|^{2} .
$$


Proof. By (4.2)-(4.6), we get

$$
\begin{aligned}
& (H(\varphi), \varphi)_{E_{0}} \\
= & \left(\varepsilon A^{\frac{m}{2}} u-A^{\frac{m}{2}} v, A^{\frac{m}{2}} u\right)+\left(-\varepsilon v+A^{m} v+\varepsilon^{2} u+(1-\varepsilon) A^{m} u, v\right) \\
= & \varepsilon\left\|A^{\frac{m}{2}} u\right\|^{2}-\varepsilon\|v\|^{2}+\left\|A^{\frac{m}{2}} v\right\|^{2}+\varepsilon^{2}(u, v)-\varepsilon\left(A^{\frac{m}{2}} u, A^{\frac{m}{2}} v\right) .
\end{aligned}
$$

By using hölder inequality, Young's inequality and Poincaré inequality, we deal with the terms in (4.8) by as follows:

$$
\begin{gathered}
\varepsilon^{2}(u, v) \geq-\frac{\varepsilon^{2}}{2}\|u\|^{2}-\frac{\varepsilon^{2}}{2}\|v\|^{2} \geq-\frac{\varepsilon^{2}}{2 \lambda_{1}{ }^{m}}\left\|A^{\frac{m}{2}} u\right\|^{2}-\frac{\varepsilon^{2}}{2}\|v\|^{2}, \\
-\varepsilon\left(A^{\frac{m}{2}} u, A^{\frac{m}{2}} v\right) \geq-\frac{\varepsilon}{2}\left\|A^{\frac{m}{2}} u\right\|^{2}-\frac{\varepsilon}{2}\left\|A^{\frac{m}{2}} v\right\|^{2} .
\end{gathered}
$$

By $0<\varepsilon<\min \left\{1, \frac{\lambda_{1}{ }^{m}}{2}, \frac{-\frac{5}{2}-\lambda_{1}{ }^{m}+\sqrt{\left(\frac{5}{2}+\lambda_{1}^{m}\right)^{2}+4 \lambda_{1}^{m}}}{2}\right\}$ and substituting (4.9)-(4.10) into (4.8), we obtain

$$
\begin{aligned}
& (H(\varphi), \varphi)_{E_{0}} \\
\geq & \left(\frac{\varepsilon}{2}-\frac{\varepsilon^{2}}{2 \lambda_{1}{ }^{m}}\right)\left\|A^{\frac{m}{2}} u\right\|^{2}+\left(\frac{1}{2}-\frac{\varepsilon}{2}\right)\left\|A^{\frac{m}{2}} v\right\|^{2}+\left(-\frac{\varepsilon^{2}}{2}-\varepsilon\right)\|v\|^{2}+\frac{1}{2}\left\|A^{\frac{m}{2}} v\right\|^{2} \\
\geq & \frac{\varepsilon}{4}\left\|A^{\frac{m}{2}} u\right\|^{2}+\left(\frac{\lambda_{1}{ }^{m}}{2}-\frac{\varepsilon \lambda_{1}{ }^{m}}{2}-\varepsilon-\frac{\varepsilon^{2}}{2}\right)\|v\|^{2}+\frac{1}{2}\left\|A^{\frac{m}{2}} v\right\|^{2} \\
\geq & \frac{\varepsilon}{4}\left(\left\|A^{\frac{m}{2}} u\right\|^{2}+\|v\|^{2}\right)+\frac{1}{2}\left\|A^{\frac{m}{2}} v\right\|^{2} \\
= & \frac{\varepsilon}{4}\|\varphi\|_{E_{0}}^{2}+\frac{1}{2}\left\|A^{\frac{m}{2}} v\right\|^{2} .
\end{aligned}
$$

The proof of Lemma 4.1.1 is completed.

The linearized equations of (1.1)-(1.3), the above equations as follows:

$$
\begin{gathered}
U_{t t}+A^{m} U_{t}+\left(\alpha+\beta\left\|A^{\frac{m}{2}} u\right\|^{2}\right)^{q} A^{m} U \\
+2 q \beta\left(\alpha+\beta\left\|A^{\frac{m}{2}} u\right\|^{2}\right)^{q-1}\left(A^{\frac{m}{2}} U, A^{\frac{m}{2}} u\right) A^{m} u+g^{\prime}(u) U=0, \\
\left.U(x, t)\right|_{x \in \partial \Omega}=0, t>0, \\
U(x, 0)=\xi, U_{t}(x, 0)=\zeta,
\end{gathered}
$$

where $(\xi, \zeta) \in E_{0},\left(u, u_{t}\right)=S(t)\left(u_{0}, u_{1}\right)$ is the solution of (1.1)-(1.3) with $\left(u_{0}, u_{1}\right) \in \mathcal{A}$.

Given $\left(u_{0}, u_{1}\right) \in \mathcal{A}$ and $S(t): E_{0} \rightarrow E_{0}$, the solution $S(t)\left(u_{0}, u_{1}\right) \in E_{0}$, by stand methods we can show that for any $(\xi, \zeta) \in$ $E_{0}$, the linear initial boundary value problem (4.12)-(4.14) possess a unique solution $\left(U(t), U_{t}(t)\right) \in L^{\infty}\left((0,+\infty) ; E_{0}\right)$.

Lemma 4.1.2. For any $t>0, R>0$, the mapping $S(t): E_{0} \rightarrow E_{0}$ is Fréchet differentiable on. Its differential at $\varphi=\left(u_{0}, u_{1}\right)^{T}$ is the linear operator on $F:(\xi, \zeta)^{T} \rightarrow(U(t), V(t))^{T}$, where $U(t)$ is the solution of (4.12)-(4.14).

Proof. Let $\varphi_{0}=\left(u_{0}, u_{1}\right)^{T} \in E_{0}, \tilde{\varphi}_{0}=\left(u_{0}+\xi, u_{1} \zeta\right)^{T} \in E_{0}$ with $\left\|\varphi_{0}\right\|_{E_{0}} \leq R,\left\|\tilde{\varphi}_{0}\right\|_{E_{0}} \leq R$, we denote $\left(u, u_{t}\right)^{T}=$ $S(t) \varphi_{0},\left(\tilde{u}, \tilde{u}_{t}\right)^{T}=S(t) \tilde{\varphi}_{0}$. We can get the Lipchitz property of $S(t)$ on the bounded sets of $E_{0}$, that is

$$
\left\|S(t) \varphi_{0}-S(t) \tilde{\varphi}_{0}\right\|_{E_{0}}^{2} \leq e^{C_{10} t}\|(\xi, \zeta)\|_{E_{0}}^{2}
$$

Let $\theta=\tilde{u}-u-U$ is the solution of problem

$$
\theta_{t t}+A^{m} \theta_{t}+\left(\alpha+\beta\left\|A^{\frac{m}{2}} u\right\|^{2}\right)^{q} A^{m} \theta=h
$$




$$
\theta(0)=\theta_{t}(0)=0
$$

with

$$
\begin{aligned}
h= & {\left[\left(\alpha+\beta\left\|A^{\frac{m}{2}} u\right\|^{2}\right)^{q}-\left(\alpha+\beta\left\|A^{\frac{m}{2}} \tilde{u}\right\|^{2}\right)^{q}\right] A^{m} \tilde{u} } \\
& +2 q \beta\left(\alpha+\beta\left\|A^{\frac{m}{2}} u\right\|^{2}\right)^{q-1}\left(A^{\frac{m}{2}} U, A^{\frac{m}{2}} u\right) A^{m} u+g(u)-g(\tilde{u})+g^{\prime}(u) U .
\end{aligned}
$$

Taking the scalar product of each side of (4.16) with $\theta_{t}$. Because of

$$
\begin{aligned}
& \left(\left[\left(\alpha+\beta\left\|A^{\frac{m}{2}} u\right\|^{2}\right)^{q}-\left(\alpha+\beta\left\|A^{\frac{m}{2}} \tilde{u}\right\|^{2}\right)^{q}\right] A^{m} \tilde{u}+2 q \beta\left(\alpha+\beta\left\|A^{\frac{m}{2}} u\right\|^{2}\right)^{q-1}\left(A^{\frac{m}{2}} U, A^{\frac{m}{2}} u\right) A^{m} u, \theta_{t}\right) \\
= & \left(\left[\left(\alpha+\beta\left\|A^{\frac{m}{2}} u\right\|^{2}\right)^{q}-\left(\alpha+\beta\left\|A^{\frac{m}{2}} \tilde{u}\right\|^{2}\right)^{q}\right] A^{m} \tilde{u}, \theta_{t}\right) \\
& +\left(2 q \beta\left(\alpha+\beta\left\|A^{\frac{m}{2}} u\right\|^{2}\right)^{q-1}\left(A^{\frac{m}{2}}(u-\tilde{u}-\theta), A^{\frac{m}{2}} u\right) A^{m} u, \theta_{t}\right) \\
\leq & C_{11}\left(R_{0}\right)\left\|A^{\frac{m}{2}} u-A^{\frac{m}{2}} \tilde{u}\right\|\left\|A^{\frac{m}{2}} \theta_{t}\right\|+C_{12}\left(R_{0}\right)\left\|A^{\frac{m}{2}} \theta\right\|\left\|A^{\frac{m}{2}} \theta_{t}\right\| .
\end{aligned}
$$

By $\left(H_{5}\right)$, we have

$$
\begin{aligned}
& \left(g(u)-g(\tilde{u})+g^{\prime}(u) U, \theta_{t}\right) \\
= & \left(g(u)-g(\tilde{u})-g^{\prime}(u)(u-\tilde{u})-g^{\prime}(u) \theta, \theta_{t}\right) \\
\leq & C_{12}\left(R_{0}\right)\left\|A^{\frac{m}{2}} u-A^{\frac{m}{2}} \tilde{u}\right\|^{1+\delta_{1}}\left\|\theta_{t}\right\|+C_{13}\left(R_{0}\right)\left\|A^{\frac{m}{2}} \theta\right\|\left\|A^{\frac{m}{2}} \theta_{t}\right\|
\end{aligned}
$$

By (4.19)-(4.20) and Young's inequality, we have

$$
\begin{aligned}
& \frac{d}{d t}\left[\left\|\theta_{t}\right\|^{2}+\left(\alpha+\beta\left\|A^{\frac{m}{2}} u\right\|^{2}\right)^{q}\left\|A^{\frac{m}{2}} \theta\right\|^{2}\right] \\
\leq & C_{14}\left[\left\|\theta_{t}\right\|^{2}+\left(\alpha+\beta\left\|A^{\frac{m}{2}} u\right\|^{2}\right)^{q}\left\|A^{\frac{m}{2}} \theta\right\|^{2}\right]+C_{15}\left(\left\|A^{\frac{m}{2}} u-A^{\frac{m}{2}} \tilde{u}\right\|^{2}+\left\|A^{\frac{m}{2}} u-A^{\frac{m}{2}} \tilde{u}\right\|^{2+2 \delta_{1}}\right) .
\end{aligned}
$$

By the Gronwall's inequality and (4.15), we get

$$
\begin{aligned}
& \left\|\theta_{t}\right\|^{2}+\left\|A^{\frac{m}{2}} \theta\right\|^{2} \\
\leq & C_{16} e^{C_{17} t} \int_{0}^{t}\left(\left\|A^{\frac{m}{2}} u-A^{\frac{m}{2}} \tilde{u}\right\|^{2}+\left\|A^{\frac{m}{2}} u-A^{\frac{m}{2}} \tilde{u}\right\|^{2+2 \delta_{1}}\right) d \tau \\
\leq & C_{18} e^{C_{19} t}\left[\left(\left\|A^{\frac{m}{2}} \xi\right\|^{2}+\|\zeta\|^{2}\right)+\left(\left\|A^{\frac{m}{2}} \xi\right\|^{2}+\|\zeta\|^{2}\right)^{1+\delta_{1}}\right],
\end{aligned}
$$

where $C_{16}, C_{17}, C_{18}, C_{19}>0$.

From (4.22), we obtain

$$
\begin{gathered}
\frac{\|\tilde{\varphi}(t)-\varphi(t)-U(t)\|_{E_{0}}^{2}}{\left\|(\xi, \zeta)^{T}\right\|_{E_{0}}^{2}} \\
\leq C_{18} e^{C_{19} t}\left[\left(\left\|A^{\frac{m}{2}} \xi\right\|^{2}+\|\zeta\|^{2}\right)+\left(\left\|A^{\frac{m}{2}} \xi\right\|^{2}+\|\zeta\|^{2}\right)^{1+\delta_{1}}\right] \rightarrow 0,
\end{gathered}
$$

as $(\xi, \zeta)^{T} \rightarrow 0$ in $E_{0}$. The proof is competed.

\subsection{The Upper Bounds of Hausdorff Dimensions for the Global Attractor}

Consider the first variation of (4.4) with initial condition:

$$
\Psi_{t}^{\prime}+P(\varphi) \Psi=\Gamma_{1}(\varphi) \Psi+\Gamma_{2}(\varphi) \Psi, \Psi(0)=(\xi, \zeta)^{T} \in E_{0}, t>0,
$$


where $\Psi=(U, V)^{T} \in E_{0}, V=U_{t}+\varepsilon U$ and $\varphi=(u, v)^{T} \in E_{0}$ is a solution of (4.3),

$$
\begin{gathered}
P(\varphi)=\left[\begin{array}{cc}
\varepsilon I & -I \\
\varepsilon^{2} I+(1-\varepsilon) A^{m} & -\varepsilon I+A^{m}
\end{array}\right], \\
\Gamma_{1}(\varphi)=\left[\begin{array}{cc}
0 & 0 \\
-g^{\prime}(u) & 0
\end{array}\right], \\
\Gamma_{2}(\varphi)=\left[\left[1-\left(\alpha+\beta\left\|A^{\frac{m}{2}} u\right\|^{2}\right)^{q}\right] A^{m} U-2 q \beta\left(\alpha+\beta\left\|A^{\frac{m}{2}} u\right\|^{2}\right)^{q-1}\left(A^{\frac{m}{2}} U, A^{\frac{m}{2}} u\right) A^{m} u\right] .
\end{gathered}
$$

It is easy to show from Lemma 4.1.2 that (4.24) is a well-posed problem in $E_{0}$, the mapping $S_{\varepsilon}(\tau):\left\{u_{0}, v_{1}=u_{1}+\varepsilon u_{0}\right\} \longrightarrow$ $\left\{u(\tau), v(\tau)=u_{t}(\tau)+\varepsilon u(\tau)\right\}, \psi(\tau)=\left\{u(\tau), v_{t}(\tau)=u_{t}(\tau)+\varepsilon u(\tau)\right\}$ is Fréchet differentiable on $E_{0}$ for any $t \geq 0$, its differential at $\varphi=\left(u_{0}, u_{1}+\varepsilon u_{0}\right)^{T}$ is the linear operator on $E_{0},(\xi, \zeta)^{T} \rightarrow(U(t), V(t))^{T}$, where $(U(t), V(t))^{T}$ is the solution of (4.24).

Lemma 4.2.1. ${ }^{(T e m a n, R .1998)}$ For any orthonormal family of elements of $\left(E_{0},\|\|_{E_{0}}\right),\left(\xi_{j}, \zeta_{j}\right)^{T}, j=1,2, \cdots, n_{1}$, we have

$$
\sum_{j=1}^{n_{1}}\left\|A^{\frac{m}{2} v} \xi_{j}\right\|^{2} \leq 2 \sum_{j=1}^{n_{1}} \mu_{j}^{v-1}, v \in[0,1),
$$

where $\left\{\mu_{j}\right\}_{j=1}^{+\infty}$ is the eigenvalue of $A^{m}$.

Proof. This is a direct consequence of Lemma VI 6.3 of [17].

Theorem 4.2.2. If we take proper $\alpha, \beta$ satisfies $\frac{1+\left(\alpha+\beta R_{0}^{2}\right)^{q}+2 q \beta R_{0}{ }^{2}\left(\alpha+\beta R_{0}{ }^{2}\right)^{q-1}}{2}-\frac{\varepsilon}{8} \leq 0$ and $\left(H_{1}\right)-\left(H_{5}\right)$ hold, then there exists $\rho\left(R_{0}\right)>0$, such that the Hausdorff dimension of global attractor $\mathcal{A}$ in $E_{0}$ satisfies

$$
d_{H}(\mathcal{A}) \leq \min \left\{n_{1} \mid n_{1} \in N, \frac{1}{n_{1}} \sum_{j=1}^{n_{1}} \mu_{j}^{\delta-1}<\frac{\varepsilon}{8 \rho n_{1}}\right\},
$$

where $R_{0}$ is as in Lemma 2.4, and

$$
\delta=\left\{\begin{array}{c}
\frac{(n-2)(p-1)-2}{2}, \frac{n}{n-2 m} \leq p<\frac{n+2 m}{n-2 m}, n \geq 2 m, \\
0, n<2 m \quad \text { or } \quad 0 \leq p \leq \frac{n}{n-2 m}, n \geq 2 m .
\end{array}\right.
$$

Proof. Let $n_{1} \in N$ be fixed. Consider $m_{1}$ solutions $\Psi_{1}, \Psi_{2}, \cdots, \Psi_{n_{1}}$ of (4.24). At a given time $\tau$, let $Q_{n_{1}}(\tau)$ denote the orthogonal projection in $E_{0}$ onto $\operatorname{span}\left\{\Psi_{1}(s), \Psi_{2}(s), \cdots, \Psi_{n_{1}}(s)\right\}$. Let $y_{j}(s)=\left(\xi_{j}, \zeta_{j}\right)^{T} \in E_{0}, j=1,2, \cdots, n_{1}$, be an orthonormal basis of

$$
Q_{n_{1}}(s) E_{0}=\operatorname{span}\left\{\Psi_{1}(s), \Psi_{2}(s), \cdots, \Psi_{n_{1}}(s)\right\},
$$

with respect to the inner product $(,)_{E_{0}}$ and norm $|\||_{E_{0}}$.

Suppose

$$
\varphi(\tau)=(u(\tau), v(\tau))^{T} \in \mathcal{A},
$$

then $\|\varphi(\tau)\|_{E_{0}} \leq M_{0}, \forall s \geq \tau$. By $\left\|y_{j}\right\|_{E_{0}}=1$ and Lemma 4.1.1, we have

$$
\begin{gathered}
-\left(P(\varphi(s)) y_{j}(s), y_{j}(s)\right)_{E_{0}} \leq-\frac{\varepsilon}{4}-\frac{1}{2}\left\|A^{\frac{m}{2}} \zeta_{j}\right\|^{2} . \\
\left(\Gamma_{1}(\varphi(s)) y_{j}(s), y_{j}(s)\right)_{E_{0}} \leq\left\|A^{-\frac{m}{2}} g^{\prime}(u) \xi_{j}(s)\right\|\left\|A^{\frac{m}{2}} \zeta_{j}(s)\right\| .
\end{gathered}
$$

By the hypothesis $\left(H_{4}\right)$, the mean value theorem and the Sobolev embedding theorem:

$$
H_{0}^{m v}(\Omega) \subset D\left(A^{\frac{m}{2} v}\right) \subset H^{m v}(\Omega) \subset L^{q}(\Omega) \subset L^{2}(\Omega) \subset L^{q^{\prime}}(\Omega) \subset H^{-m v}(\Omega),
$$


where $\frac{1}{q}=\frac{1}{2}-\frac{m v}{n}, \frac{1}{q}+\frac{1}{q^{\prime}}=1, v \in[0,1]$.

So, by Lemma 2.4 and (4.34), for $n=1, H_{0}^{m}(\Omega) \subset L^{\infty}(\Omega) \subset L^{1}(\Omega) \subset H^{-m}(\Omega) \subset\left(H_{0}^{m}(\Omega)\right)^{\prime}$. There exists $C_{2} 0\left(R_{0}\right)>0$, we get

$$
\left\|A^{-\frac{m}{2}}\left(g^{\prime}(u) \xi_{j}(s)\right)\right\| \leq C_{2} 0\left\|g^{\prime}(u) \xi_{j}(s)\right\|_{L^{1}} \leq C_{21}\left(R_{0}\right)\left\|\xi_{j}(s)\right\| .
$$

For $1<n \leq 2 m, H_{0}^{m}(\Omega) \subset L^{q}(\Omega) \subset H^{-m}(\Omega) \subset\left(H_{0}^{m}(\Omega)\right)^{\prime}, q>0$, there exists $C_{11}\left(R_{0}\right)>0$, such that

$$
\left\|A^{-\frac{m}{2}}\left(g^{\prime}(u) \xi_{j}(s)\right)\right\| \leq C_{9}\left\|g^{\prime}(u) \xi_{j}(s)\right\|_{L^{\frac{3}{2}}} \leq C_{11}\left(R_{0}\right)\left\|\xi_{j}(s)\right\| .
$$

For $n>2 m$, by $\left(H_{4}\right)$, there exists $C_{22}\left(R_{0}\right)>0$, such that

$$
\left\|A^{-\frac{m}{2}}\left(g^{\prime}(u) \xi_{j}(s)\right)\right\| \leq\left\|g^{\prime}(u) \xi_{j}(s)\right\|_{L^{n+2 m}} \leq C_{22}\left(R_{0}\right)\left\|A^{\frac{m}{2} \delta} \xi_{j}(s)\right\| .
$$

From (4.34)-(4.38), we have

$$
\left(\Gamma_{1}(\varphi(s)) y_{j}(s), y_{j}(s)\right)_{E_{0}} \leq \frac{C_{23}}{2}\left\|A^{\frac{m}{2} \delta} \xi_{j}(s)\right\|\left\|A^{\frac{m}{2}} \zeta_{j}(s)\right\|,
$$

where $C_{23}=C_{23}\left(R_{0}\right)=2 \max \left\{C_{21}\left(R_{0}\right), C_{22}\left(R_{0}\right)\right\}$.

By Lemma 2.4, we obtain

$$
\begin{aligned}
& \left(\Gamma_{2}(\varphi(s)) y_{j}(s), y_{j}(s)\right) \\
= & {\left[1-\left(\alpha+\beta\left\|A^{\frac{m}{2}} u\right\|^{2}\right)^{q}\right]\left(A^{\frac{m}{2}} \xi_{j}, A^{\frac{m}{2}} \zeta_{j}\right)-2 q \beta\left(\alpha+\beta\left\|A^{\frac{m}{2}} u\right\|^{2}\right)^{q-1}\left(A^{\frac{m}{2}} \xi_{j}, A^{\frac{m}{2}} u\right)\left(A^{\frac{m}{2}} u, A^{\frac{m}{2}} \zeta_{j}\right) } \\
\leq & {\left[1+\left(\alpha+\beta R_{0}^{2}\right)^{q}+2 q \beta R_{0}{ }^{2}\left(\alpha+\beta R_{0}^{2}\right)^{q-1}\right]\left\|A^{\frac{m}{2}} \xi_{j}\right\|\left\|A^{\frac{m}{2}} \zeta_{j}\right\| . }
\end{aligned}
$$

By lemma VI 6.3 of [17], Young's inequality and choose $\alpha, \beta$ satisfying $\frac{1+\left(\alpha+\beta R_{0}^{2}\right)^{q}+2 q \beta R_{0}^{2}\left(\alpha+\beta R_{0}^{2}\right)^{q-1}}{2}-\frac{\varepsilon}{8} \leq 0$, we obtain

$$
\begin{aligned}
p_{n_{1}}(s) & =\sum_{j=1}^{n_{1}}\left(\left(-P(\varphi(s))+\Gamma_{2}\left(\varphi(s)+\Gamma_{1}(\varphi(s))\right) y_{j}(s), y_{j}(s)\right)_{E_{0}}\right. \\
& \leq\left[\frac{1+\left(\alpha+\beta R_{0}^{2}\right)^{q}+2 q \beta R_{0}^{2}\left(\alpha+\beta R_{0}^{2}\right)^{q-1}}{2}-\frac{\varepsilon}{4}\right] n_{1}+\frac{\rho}{8}\left\|A^{\frac{m}{2} \delta} \xi_{j}\right\| \\
& \leq-\frac{\varepsilon}{8} n_{1}+\frac{\rho}{2} \sum_{j=1}^{n_{1}} \mu_{j}^{\delta-1}
\end{aligned}
$$

where $\rho=C_{23}{ }^{2}\left(R_{0}\right)$

If $\frac{\varepsilon}{8 \rho n_{1}} \geq \frac{1}{n_{1}} \sum_{j=1}^{n_{1}} \lambda_{j}^{\delta-1}$, then

$$
q_{n_{1}}=\lim _{t \rightarrow \infty} \inf \sup _{\tau \in R} \sup _{\Phi \subset E_{0}} \sup _{\varphi(\tau) \in \mathcal{A}} \frac{1}{t} \int_{\tau}^{\tau+t} p_{n_{1}}(s) d s \leq-\rho n_{1}\left(\frac{\varepsilon}{4 \rho n_{1}}-\frac{1}{n_{1}} \sum_{j=1}^{n_{1}} \lambda_{j}^{\delta-1}\right)<0 .
$$

So, by lemma 4 of (S. Zhou, 1999), we obtain (4.29). The proof of Theorem 4.2.2 is completed.

\section{Exponential Attractor}

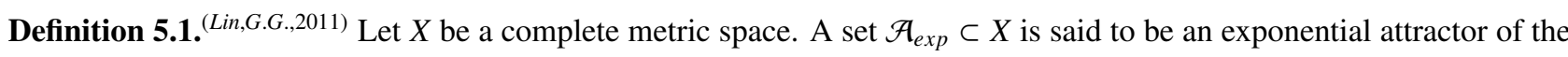
dynamical system $(S(t), X)$ if

(i) it is a compact set in $X$; 
(ii) it has finite fractal dimension in $X$, i.e. $\operatorname{dim}_{f}\left\{\mathcal{A}_{\text {exp }}, X\right\}<+\infty$;

(iii) it is a forward invariant set, i.e. $S(t) \mathcal{A}_{\text {exp }} \subset \mathcal{A}_{\text {exp }}, \quad t \geq 0$;

(iv) it attractor exponentially the bounded sets in $X$, that is, for any bounded $\operatorname{set} B \subset X$, there exists a positive constant $k$ such that

$$
\operatorname{dist}_{X}\left(S(t) B, \mathcal{A}_{\exp }\right) \leq C\left(\|B\|_{X}\right) e^{-k t}, \quad t \geq 0
$$

where $\|B\|_{X}=\sup _{\zeta \in B}\|\zeta\|_{X}$.

Lemma 5.2.(Interpolation theorem ${ }^{\text {(RobertA.Adams\&JohnJ.F.Fournier,2003) }}$ ) Let $1 \leq p<q<r$, so that

$$
\frac{1}{q}=\frac{\theta}{p}+\frac{1-\theta}{r}
$$

for some $\theta$ satisfying $0<\theta<1$. If $u \in L^{p}(\Omega) \cap L^{r}(\Omega)$, then $u \in L^{q}(\Omega)$ and

$$
\|u\|_{q} \leq\|u\|_{p}^{\theta}\|u\|_{r}^{1-\theta} .
$$

Lemma 5.3. ${ }^{\text {(ZhijianYang\&PengyanDing.,2016) }}$ Let $y: R^{+} \rightarrow R^{+}$be an absolutely continuous function satisfying

$$
\frac{d}{d t} y(t)+2 \epsilon y(t) \leq h(t) y(t)+z(t), \quad t>0,
$$

where $\epsilon>0, z \in L_{l o c}^{1}\left(R^{+}\right), \int_{s}^{t} h(\tau) d \tau \leq \epsilon(t-s)+m$ for $t \geq s \geq 0$ and some $m>0$. Then

$$
y(t) \leq e^{m}\left(y(0) e^{-\epsilon t}+\int_{0}^{t}|z(\tau)| e^{-\epsilon(t-\tau)} d \tau\right), \quad t>0 .
$$

Lemma 5.4. Under assumptions of Lemma 2.4 and Lemma 2.5, there exist $t_{2}, R_{2} \geq 0$, such that

$$
\left\|u_{t t}\right\|^{2} \leq R_{2}^{2}, \quad t \geq t_{2}
$$

Proof. Differentiate to (1.1) about $t$, we get

$$
\begin{aligned}
& u_{t t t}+(-\Delta)^{m} u_{t t}+\left(\alpha+\beta\left\|\nabla^{m} u\right\|^{2}\right)^{q}(-\Delta)^{m} u_{t} \\
& +2 q \beta\left(\alpha+\beta\left\|\nabla^{m} u\right\|^{2}\right)^{q-1}\left(\nabla^{m} u, \nabla^{m} u_{t}\right)(-\Delta)^{m} u+g^{\prime}(u) u_{t}=0, \\
& u(x, 0)=u_{0}(x), u_{t}(x, 0)=u_{1}(x), \\
& u_{t t}(x, 0)=f(x)-(-\Delta)^{m} u_{1}(x)-\left(\alpha+\beta\left\|\nabla^{m} u_{0}\right\|^{2}\right)^{q}(-\Delta)^{m} u_{0}-g\left(u_{0}\right), \\
& u(x, t)=0, \frac{\partial^{i} u}{\partial \nu^{i}}=0, i=1,2, \cdots, m-1, x \in \partial \Omega, t \in(0,+\infty) .
\end{aligned}
$$

Now we use the multiplier $u_{t t}$ for (5.7). We readily obtain

$$
\begin{aligned}
& \frac{d}{d t}\left[\left\|u_{t t}\right\|^{2}+\left(\alpha+\beta\left\|\nabla^{m} u\right\|^{2}\right)^{q}\left\|\nabla^{m} u_{t}\right\|^{2}\right]+2\left\|\nabla^{m} u_{t t}\right\|^{2} \\
& =2 q \beta\left(\alpha+\beta\left\|\nabla^{m} u\right\|^{2}\right)^{q-1}\left(\nabla^{m} u, \nabla^{m} u_{t}\right)\left[\left\|\nabla^{m} u_{t}\right\|^{2}-2\left((-\Delta)^{m} u, u_{t t}\right)\right]-2\left(g^{\prime}(u) u_{t}, u_{t t}\right) .
\end{aligned}
$$

By Lemma 2.4 and Lemma 2.5, we get

$$
\begin{gathered}
2 q \beta\left(\alpha+\beta\left\|\nabla^{m} u\right\|^{2}\right)^{q-1}\left(\nabla^{m} u, \nabla^{m} u_{t}\right)\left\|\nabla^{m} u_{t}\right\|^{2}<C_{24}\left(R_{0}, R_{1}\right), \quad(t \geq 0), \\
4 q \beta\left(\alpha+\beta\left\|\nabla^{m} u\right\|^{2}\right)^{q-1}\left(\nabla^{m} u, \nabla^{m} u_{t}\right)\left\|(-\Delta)^{m} u\right\|<C_{25}\left(R_{0}, R_{1}\right), \quad(t \geq 0), \\
2\left\|g^{\prime}(u) u_{t}\right\|<C_{26}\left(R_{0}, R_{1}\right), \quad(t \geq 0),
\end{gathered}
$$




$$
2\left\|\nabla^{m} u_{t t}\right\|^{2} \geq 2 \lambda_{1}{ }^{m}\left\|u_{t t}\right\|^{2} .
$$

From (5.8)-(5.12) and Young's inequality, we have

$$
\begin{aligned}
& \frac{d}{d t}\left[\left\|u_{t t}\right\|^{2}+\left(\alpha+\beta\left\|\nabla^{m} u\right\|^{2}\right)^{q}\left\|\nabla^{m} u_{t}\right\|^{2}\right]+2 \lambda_{1}{ }^{m}\left\|u_{t t}\right\|^{2} \\
\leq & C_{27}+C_{25}\left\|u_{t t}\right\|^{2} \\
\leq & C_{27}+\frac{C_{25}}{4 \varepsilon}+\varepsilon\left\|u_{t t}\right\|^{2},
\end{aligned}
$$

where $C_{24}, C_{25}, C_{26}>0$ and $C_{27}=C_{24}+C_{26}$.

We choose $\varepsilon<2 \lambda_{1}{ }^{m}$ and Lemma 2.5, then

$$
\begin{aligned}
& \frac{d}{d t}\left[\left\|u_{t t}\right\|^{2}+\left(\alpha+\beta\left\|\nabla^{m} u\right\|^{2}\right)^{q}\left\|\nabla^{m} u_{t}\right\|^{2}\right]+\left(2 \lambda_{1}{ }^{m}-\varepsilon\right)\left[\left\|u_{t t}\right\|^{2}+\left(\alpha+\beta\left\|\nabla^{m} u\right\|^{2}\right)^{q}\left\|\nabla^{m} u_{t}\right\|^{2}\right] \\
& \leq C_{27}+\frac{C_{25}{ }^{2}}{4 \varepsilon}+\left(2 \lambda_{1}{ }^{m}-\varepsilon\right)\left(\alpha+\beta\left\|\nabla^{m} u\right\|^{2}\right)^{q}\left\|\nabla^{m} u_{t}\right\|^{2} \\
& \leq C_{28} .
\end{aligned}
$$

By Gronwall's inequality for (5.14), we obtain

$$
\begin{aligned}
& \left\|u_{t t}\right\|^{2}+\left(\alpha+\beta\left\|\nabla^{m} u\right\|^{2}\right)^{q}\left\|\nabla^{m} u_{t}\right\|^{2} \\
\leq & {\left[\left\|u_{t t}(x, 0)\right\|^{2}+\left(\alpha+\beta\left\|\nabla^{m} u_{0}\right\|^{2}\right)^{q}\left\|\nabla^{m} u_{1}\right\|^{2}\right] e^{-\left(2 \lambda_{1}^{m}-\varepsilon\right) t}+\frac{C_{28}}{2 \lambda_{1}{ }^{m}-\varepsilon} . }
\end{aligned}
$$

Therefore, it exists $t_{2}, R_{2}>0$, such that

$$
\left\|u_{t t}\right\|^{2} \leq R_{2}^{2}, \quad t \geq t_{2} .
$$

Lemma 5.5. One of the following requirements fulfills:

Case(I): When $n>2 m$, with $1 \leq p<\frac{n+2 m}{n-2 m}$;

Case(II): When $2 m<n \leq 6 m, g \in C^{2}(R)$ is critical, such that

$$
\left|g^{\prime \prime}(u)\right| \leq C\left(1+|u|^{\frac{6 m-n}{n-2 m}}\right), u \in R
$$

Then, the following Lipschitz continuity holds:

$$
\left\|z_{t}\right\|^{2}+\left\|\nabla^{m} z\right\|^{2} \leq C\left(\left\|z_{1}\right\|^{2}+\left\|\nabla^{m} z_{0}\right\|^{2}\right) e^{-k t}+C \int_{0}^{t} e^{-k(t-\tau)}\|z(\tau)\|^{2} d \tau
$$

where $k>0, z=u-v . u, v$ are the solutions of problem (1.1)-(1.3) corresponding to initial data $\left(u_{0}, u_{1}\right)$ and $\left(v_{0}, v_{1}\right)$ in $H_{0}^{m}(\Omega) \times L^{2}(\Omega)$.

Proof. Obviously, we have

$$
\begin{aligned}
& z_{t t}+(-\Delta)^{m} z_{t}+M(t)(-\Delta)^{m} z+\bar{M}(t)\left(\nabla^{m}(u+v), \nabla^{m} z\right)(-\Delta)^{m}(u+v)+f(u)-f(v)=0, \\
& z(0)=u_{0}-v_{0}, z_{t}(0)=u_{1}-v_{1},
\end{aligned}
$$

where

$$
\begin{gathered}
M(t)=\frac{1}{2}\left[\left(\alpha+\beta\left\|\nabla^{m} u\right\|^{2}\right)^{q}+\left(\alpha+\beta\left\|\nabla^{m} v\right\|^{2}\right)^{q}\right] \geq \alpha^{q}, \\
\bar{M}(t)=\frac{1}{2} \int_{0}^{1} q \beta\left[\alpha+\beta\left(\lambda\left\|\nabla^{m} u\right\|^{2}+(1-\lambda)\left\|\nabla^{m} v\right\|^{2}\right)\right]^{q-1} d \lambda \geq 0 .
\end{gathered}
$$


By multiplying (5.19) by $z_{t}$ and Lemma 2.4, Lemma 2.5, we get

$$
\begin{aligned}
& \frac{d}{d t}\left[\left\|z_{t}\right\|^{2}+M(t)\left\|\nabla^{m} z\right\|^{2}+\bar{M}(t)\left(\nabla^{m}(u+v), \nabla^{m} z\right)^{2}\right]+2\left\|\nabla^{m} z_{t}\right\|^{2} \\
= & {\left[q \beta\left(\alpha+\beta\left\|\nabla^{m} u\right\|^{2}\right)^{q-1}\left(\nabla^{m} u, \nabla^{m} u_{t}\right)+q \beta\left(\alpha+\beta\left\|\nabla^{m} v\right\|^{2}\right)^{q-1}\left(\nabla^{m} v, \nabla^{m} v_{t}\right)\right]\left\|\nabla^{m} z\right\|^{2} } \\
& +2 \bar{M}(t)\left(\nabla^{m}\left(u_{t}+v_{t}\right), \nabla^{m} z\right)\left(\nabla^{m}(u+v), \nabla^{m} z\right) \\
& +\int_{0}^{1} q(q-1) \beta^{2}\left(\alpha+\beta \lambda\left\|\nabla^{m} u\right\|^{2}+\beta(1-\lambda)\left\|\nabla^{m} v\right\|^{2}\right)^{q-2} \\
& \times\left(\lambda\left(\nabla^{m} u, \nabla^{m} u_{t}\right)+(1-\lambda)\left(\nabla^{m} v, \nabla^{m} v_{t}\right)\right) d \lambda\left(\nabla^{m}(u+v), \nabla^{m} z\right)^{2}-2\left(g(u)-g(v), z_{t}\right) \\
\leq & C_{29}\left(\left\|\nabla^{m} u_{t}\right\|+\left\|\nabla^{m} v_{t}\right\|\right)\left\|\nabla^{m} z\right\|^{2}-2\left(g(u)-g(v), z_{t}\right) .
\end{aligned}
$$

Case(I): When $1 \leq p<\frac{n+2 m}{n-2 m}$, there exists a $\delta: 1>>\delta>0$ such that $H_{0}^{m-\delta}(\Omega) \hookrightarrow L^{p+1}(\Omega)$. By the interpolation and Lemma 2.5 , we get

$$
\begin{aligned}
& -2\left(g(u)-g(v), z_{t}\right) \\
\leq & 2 C_{1} \int_{\Omega}\left(|u|^{p-1}+|v|^{p-1}\right)|z|\left|z_{t}\right| d x \\
\leq & 2 C_{1}\left(\|u\|_{p+1}^{p-1}+\|v\|_{p+1}^{p-1}\right)\|z\|_{p+1}\left\|z_{t}\right\|_{p+1} \\
\leq & 2 C_{1}\left(\|u\|_{\frac{2 n}{n+2 m}}^{\theta(p-1)}\|u\|^{(1-\theta)(p-1)}+\|v\|_{\frac{2 n}{n+2 m}}^{\theta(p-1)}\|v\|^{(1-\theta)(p-1)}\right)\|z\|_{p+1}\left\|z_{t}\right\|_{p+1} \\
\leq & C_{30}\|z\|_{H_{0}^{m-\delta}}\left\|\nabla^{m} z_{t}\right\| \\
\leq & C_{31}\|z\|^{\delta}\left\|\nabla^{m} z\right\|^{1-\delta}\left\|\nabla^{m} z_{t}\right\| \\
\leq & \varepsilon_{3}\left\|\nabla^{m} z_{t}\right\|^{2}+\varepsilon_{3}^{2}\left\|\nabla^{m} z\right\|^{2}+C_{32}\|z\|^{2},
\end{aligned}
$$

where $\theta=\frac{n(p-1)}{(p+1)(n+2 m)}, C_{30}, C_{31}, C_{32}>0$ and $2>\varepsilon_{3}>0$.

Inserting (5.23) into (5.22), we have

$$
\begin{aligned}
& \frac{d}{d t}\left[\left\|z_{t}\right\|^{2}+M(t)\left\|\nabla^{m} z\right\|^{2}+\bar{M}(t)\left(\nabla^{m}(u+v), \nabla^{m} z\right)^{2}\right]+\left(2-\varepsilon_{3}\right)\left\|\nabla^{m} z_{t}\right\|^{2} \\
\leq & \varepsilon_{3}{ }^{2}\left\|\nabla^{m} z\right\|^{2}+C_{29}\left(\left\|\nabla^{m} u_{t}\right\|+\left\|\nabla^{m} v_{t}\right\|\right)\left\|\nabla^{m} z\right\|^{2}+C_{32}\|z\|^{2} .
\end{aligned}
$$

We take the scalar product in $L^{2}$ of equation (5.19) with $z$. Then

$$
\begin{aligned}
& \frac{d}{d t}\left[\left(z_{t}, z\right)+\frac{1}{2}\left\|\nabla^{m} z\right\|^{2}\right]+M(t)\left\|\nabla^{m} z\right\|^{2}+\bar{M}(t)\left(\nabla^{m}(u+v), \nabla^{m} z\right)^{2} \\
= & \left\|z_{t}\right\|^{2}-(g(u)-g(v), z) .
\end{aligned}
$$

In (5.25), by Lemma 2.5 we have

$$
\begin{aligned}
-(g(u)-g(v), z) & \leq C_{1}\left(\|u\|_{p+1}^{p-1}+\|v\|_{p+1}^{p-1}\right)\|z\|_{p+1}^{2} \\
& \leq C_{33}\|z\|_{p+1}^{2} \\
& \leq C_{34}\|z\|_{H_{0}^{m-\delta}}^{2} \\
& \leq \varepsilon_{3}\left\|\nabla^{m} z\right\|^{2}+C_{35}\|z\|^{2},
\end{aligned}
$$

with $C_{33}, C_{34}, C_{35}>0$.

Inserting (5.25) into (5.26), we get

$$
\begin{aligned}
& \frac{d}{d t}\left[\left(z_{t}, z\right)+\frac{1}{2}\left\|\nabla^{m} z\right\|^{2}\right]+\|z\|^{2}+M(t)\left\|\nabla^{m} z\right\|^{2}+\bar{M}(t)\left(\nabla^{m}(u+v), \nabla^{m} z\right)^{2} \\
\leq & \left\|z_{t}\right\|^{2}+\varepsilon_{3}\left\|\nabla^{m} z\right\|^{2}+C_{36}\|z\|^{2},
\end{aligned}
$$

with $C_{36}=C_{35}+1$. 
Setting

$$
\begin{gathered}
P(t)=\left\|z_{t}\right\|^{2}+M(t)\left\|\nabla^{m} z\right\|^{2}+\bar{M}(t)\left(\nabla^{m}(u+v), \nabla^{m} z\right)^{2}+\varepsilon_{3}\left(\left(z_{t}, z\right)+\frac{1}{2}\left\|\nabla^{m} z\right\|^{2}\right), \\
Q(t)=\left(2-2 \varepsilon_{3}\right)\left\|\nabla^{m} z_{t}\right\|^{2}+\varepsilon_{3}\|z\|^{2}-2 \varepsilon_{3}^{2}\left\|\nabla^{m} z\right\|^{2} \\
+\varepsilon_{3} M(t)\left\|\nabla^{m} z\right\|^{2}+\varepsilon_{3} \bar{M}(t)\left(\nabla^{m}(u+v), \nabla^{m} z\right)^{2} .
\end{gathered}
$$

Obviously, there exist $a_{2} \geq a_{1}>0, k>0$ and $\varepsilon_{3}>0$ suitably small, such that

$$
\begin{gathered}
a_{1}\left[\left\|z_{t}\right\|^{2}+\left\|\nabla^{m} z\right\|^{2}+\bar{M}(t)\left(\nabla^{m}(u+v), \nabla^{m} z\right)^{2}\right] \leq P(t), \\
P(t) \leq a_{2}\left[\left\|z_{t}\right\|^{2}+\left\|\nabla^{m} z\right\|^{2}+\bar{M}(t)\left(\nabla^{m}(u+v), \nabla^{m} z\right)^{2}\right], \\
Q(t) \geq k P(t) .
\end{gathered}
$$

By $(5.24)+\varepsilon_{3} \times(5.27)$ and (5.28)-(5.32), we get

$$
\begin{aligned}
& \frac{d}{d t} P(t)+k P(t) \\
\leq & \frac{d}{d t} P(t)+Q(t) \\
\leq & C_{29}\left(\left\|\nabla^{m} u_{t}\right\|+\left\|\nabla^{m} v_{t}\right\|\right)\left\|\nabla^{m} z\right\|^{2}+\left(C_{32}+\varepsilon_{3} C_{36}\right)\|z\|^{2} \\
\leq & C_{37}\left(\left\|\nabla^{m} u_{t}\right\|+\left\|\nabla^{m} v_{t}\right\|\right)\left\|\nabla^{m} z\right\|^{2}+C_{37}\|z\|^{2},
\end{aligned}
$$

where $C_{37}=\max \left\{C_{29}, C_{32}+\varepsilon_{3} C_{36}\right\}$.

By Lemma 5.3, there exists $C>0$, we get

$$
\left\|z_{t}\right\|^{2}+\left\|\nabla^{m} z\right\|^{2} \leq C\left(\left\|z_{1}\right\|^{2}+\left\|\nabla^{m} z_{0}\right\|^{2}\right) e^{-k t}+C \int_{0}^{t} e^{-k(t-\tau)}\|z(\tau)\|^{2} d \tau
$$

Case(II): When $p=\frac{n+2 m}{n-2 m}$, we have

$$
\left(g(u)-g(v), z_{t}\right)=\frac{1}{2} \frac{d}{d t} \int_{\Omega} \int_{0}^{1}\left(g^{\prime}(\lambda u+\lambda(u-v)) z^{2} d \lambda d x+\bar{H}(t),\right.
$$

with

$$
\bar{H}(t)=-\frac{1}{2} \int_{\Omega} \int_{0}^{1} g^{\prime \prime}(\lambda u+\lambda(u-v))\left(\lambda u_{t}+(1-\lambda) v_{t}\right) z^{2} d \lambda d x .
$$

By the growth condition of $g^{\prime \prime}$, we have

$$
\bar{H}(t) \leq C \int_{\Omega}\left(1+|u|^{\frac{6 m-n}{n-2 m}}+|v|^{\frac{6 m-n}{n-2 m}}\right)\left(\left|u_{t}\right|+\left|v_{t}\right|\right)|z|^{2} d x .
$$

Therefore the Hölder inequality and $H_{0}^{m}(\Omega) \hookrightarrow L^{\frac{2 n}{n-2 m}}(\Omega)$ imply that

$$
\begin{aligned}
\bar{H}(t) & \leq C\left(1+\|u\|_{\frac{2 m}{n-2 m}}^{\frac{6 m-n}{n-2 m}}+\|v\|_{\frac{2 m}{n-2 m}}^{\frac{6 m-n}{n-2 m}}\right)\left(\left\|u_{t}\right\|_{\frac{2 n}{n-2 m}}+\left\|v_{t}\right\|_{\frac{2 n}{n-2 m}}\right)\|z\|_{\frac{2 n}{n-2 m}}^{2} \\
& \leq C_{28}\left(\left\|\nabla^{m} u_{t}\right\|+\left\|\nabla^{m} v_{t}\right\|\right)\left\|\nabla^{m} z\right\|^{2}+\varepsilon_{3}\left\|z_{t}\right\|^{2}+C_{29}\|z\|^{2}
\end{aligned}
$$

By Lemma 2.2, we get

$$
-(g(u)-g(v), z) \leq \varepsilon_{3}\left\|\nabla^{m} z\right\|^{2}+C_{30}\|z\|^{2} .
$$


So, from (5.22) and (5.27), we obtain

$$
\begin{gathered}
\bar{P}(t)=\left\|z_{t}\right\|^{2}+M(t)\left\|\nabla^{m} z\right\|^{2}+\bar{M}(t)\left(\nabla^{m}(u+v), \nabla^{m} z\right)^{2} \\
+\int_{\Omega} \int_{0}^{1} g^{\prime}(\lambda u+\lambda(u-v)) z^{2} d \lambda d x+\varepsilon_{3}\left(\left(z_{t}, z\right)+\frac{1}{2}\left\|\nabla^{m} z\right\|^{2}\right), \\
\bar{Q}(t)=2\left\|\nabla^{m} z_{t}\right\|^{2}-2 \varepsilon_{3}^{2}\left\|z_{t}\right\|^{2}+\varepsilon_{3}\|z\|^{2}+\varepsilon_{3} M(t)\left\|\nabla^{m} z\right\|^{2} \\
+\varepsilon_{3} \bar{M}(t)\left(\nabla^{m}(u+v), \nabla^{m} z\right)^{2}-\varepsilon_{3}^{2}\left\|\nabla^{m} z\right\|^{2}, \\
\frac{d}{d t} \bar{P}(t)+\bar{Q}(t) \leq\left(C_{19}+2 C_{28}\right)\left(\left\|\nabla^{m} u_{t}\right\|+\left\|\nabla^{m} v_{t}\right\|\right)\left\|\nabla^{m} z\right\|^{2}+\left(2 C_{29}+\varepsilon_{3} C_{30}\right)\|z\|^{2} .
\end{gathered}
$$

Obviously, there exist $b_{2} \geq b_{1}>0, k>0$ and $\varepsilon_{3}>0$ suitably small, such that

$$
\begin{gathered}
b_{1}\left[\left\|z_{t}\right\|^{2}+\left\|\nabla^{m} z\right\|^{2}+\bar{M}(t)\left(\nabla^{m}(u+v), \nabla^{m} z\right)^{2}\right] \leq \bar{P}(t), \\
\bar{P}(t) \leq b_{2}\left[\left\|z_{t}\right\|^{2}+\left\|\nabla^{m} z\right\|^{2}+\bar{M}(t)\left(\nabla^{m}(u+v), \nabla^{m} z\right)^{2}\right], \\
\bar{Q}(t) \geq k \bar{P}(t),
\end{gathered}
$$

where $\varepsilon_{3}>0$ is suitably small.

Therefore, Omit Case(I), we easily obtain (5.18).

Lemma 5.6. ${ }^{[28]}$ Let $X$ be a Banach space and $M$ be a bounded closed set in $X$. Assume that the mapping $V: M \rightarrow M$ possesses the properties:

(i) $V$ is Lipschitz on $M$, i.e. there exists an $L>0$ such that

$$
\left\|V v_{1}-V v_{2}\right\| \leq L\left\|v_{1}-v_{2}\right\|, \forall v_{1}, v_{2} \in M
$$

(ii) there exist compact seminorms $n_{1}(x), n_{2}(x)$ on $X$ such that

$$
\left\|V v_{1}-V v_{2}\right\| \leq \eta\left\|v_{1}-v_{2}\right\|+K\left(n_{1}\left(v_{1}-v_{2}\right)+n_{2}\left(V v_{1}-V v_{2}\right)\right)
$$

for any $v_{1}, v_{2} \in M$, where $0<\eta<1$ and $K>0$ are constants.

Then for any $k>0$ and $\delta \in(0,1-\eta)$ there exists a forward invariant compact set $A_{k, \delta} \subset M$ of finite fractal dimension such that

$$
\operatorname{dist}\left(V^{k} M, A_{k, \delta}\right) \leq q^{k}, k=1,2, \cdots,
$$

where $q=\eta+\delta<1$, and

$$
\operatorname{dim}_{f} A_{k, \delta} \leq\left[\ln \frac{1}{\delta+\eta}\right]^{-1} \cdot\left[\ln m_{0}\left(\frac{2 K\left(1+L^{2}\right)^{1 / 2}}{1-\eta}\right)+k\right],
$$

where $m_{0}(R)$ is the maximal number of pairs $\left(x_{i}, y_{i}\right)$ in $X \times X$ possessing the properties

$$
\left\|x_{i}\right\|^{2}+\left\|y_{i}\right\|^{2} \leq R^{2}, n_{1}\left(x_{i}-x_{j}\right)+n_{2}\left(y_{i}-y_{j}\right)>1, i \neq j .
$$

That is, the discrete dynamical system $\left(V^{k}, M\right)$ possesses an exponential attractor $A_{k, \delta}$.

Theorem 5.7. Let assumption of Lemma $2.4,2.5$ and 5.5 be valid, with $1 \leq p \leq \frac{n+2 m}{n-2 m}$. Then the dynamical system $\left(S(t), E_{0}\right)$ has an exponential attractor $\mathcal{A}_{\text {exp }}$.

Proof. It is proved by omitting [28]. By Theorem 3.3, we known $S(t)$ has a bounded absorbing $B_{0}$ in $E_{1}$. So $B_{0}$ is closed in $E_{2}$. From Lemma 2.4, 2.5 and Lemma 5.4, $B_{0}$ is bounded in $H_{0}^{m}(\Omega) \times H_{0}^{m}(\Omega)$, and for any $\xi_{u}=\left(u_{0}, u_{1}\right) \in B_{0}, \xi_{u}(t)=$ $S(t) \xi_{u}=\left(u(t), u_{t}(t)\right) \in B_{0}$, and

$$
\left\|\nabla^{m} u\right\|+\left\|\nabla^{m} u_{t}\right\|+\left\|u_{t t}\right\| \leq C, t \geq 0
$$


Define the operator

$$
V=S(T): B_{0} \rightarrow B_{0} .
$$

Obviously, $V B_{0} \subset B_{0}$ and $V$ is Lipschitz on $B_{0}$. For any $\xi_{u}, \xi_{v} \in B_{0}$, we infer from Lemma 5.5 that

$$
\begin{aligned}
\left\|V \xi_{u}-V \xi_{v}\right\|_{E_{0}}^{2} & \leq C e^{-k T}\left\|\xi_{u}(0)-\xi_{v}(0)\right\|_{E_{0}}^{2}+C \int_{0}^{T} e^{-k(t-\tau)}\|u(\tau)-v(\tau)\|^{2} d \tau \\
& \leq \eta_{T}^{2}\left\|\xi_{u}-\xi_{v}\right\|_{E_{0}}^{2}+C \max _{0 \leq \tau \leq T}\|u(\tau)-v(\tau)\|^{2}
\end{aligned}
$$

that is

$$
\left\|V \xi_{u}-V \xi_{v}\right\|_{E_{0}} \leq \eta_{T}\left\|\xi_{u}-\xi_{v}\right\|_{E_{0}}+C n_{1}\left(\xi_{u}-\xi_{v}\right)
$$

where $\eta_{T}^{2}=C e^{-k T}, n_{1}\left(\xi_{u}\right)=\max _{0 \leq \tau \leq T}\|u(\tau)\|$. Because of $H_{0}^{m}(\Omega) \hookrightarrow L^{2}(\Omega)$, such that $n_{1}\left(\xi_{u}\right)$ is a compact semi-norm.

By Lemma 5.6, the discrete dynamical system $\left(V^{k}, B_{0}\right)$ has an exponential attractor $\mathbb{A}$, where $V^{k}=S(k T)$. Let

$$
\mathcal{A}_{\text {exp }}=\underset{0 \leq t \leq T}{\cup} S(t) \mathbb{A} \text {. }
$$

By the standard method (Z. J. Yang, 2010), one easily knows that $\mathcal{A}_{\text {exp }}$ is an exponential attractor of dynamical system $\left(S(t), B_{0}\right)$. So there exists a $\gamma>0$ such that

$$
\operatorname{dist}_{E_{0}}\left\{S(t) B_{0}, \mathcal{A}_{\exp }\right\} \leq C e^{-\gamma t}, t \geq 0 .
$$

Similar to (Zhijian Yang \& Pengyan Ding, 2016), we easily obtain conclusion of definition 5.1. So, we obtain $\mathcal{A}_{\exp }$ is an exponential attractor of $\left(S(t), E_{0}\right)$.

\section{Acknowledgements}

The authors express their sincere thanks to the aonymous reviewer for his/her careful reading of the paper, giving valuable comments and suggestions. These contributions greatly improved the paper.

\section{References}

Ball, J. M. (1997). Remarks on blow-up and nonexistence theorems for nonlinear evolution equations. Quart. J. Math. Oxford Ser., 28(112), 473-486.

Chueshov, I., \& Lasiecka, I. (2008). Long-Time Behavior of Second Order Evolution Equations with Nonlinear Damping. Mem. Amer. Math. Soc, 195. https://doi.org/10.1090/memo/0912

Fatori, L. H., Jorge Silva, M. A., \& Ma, T. F. (2015). Long-time behavior of a class of thermoelastic plates with nonlinear strain. J. Differential Equations, 259, 4831-4862. https://doi.org/10.1016/j.jde.2015.06.026

Ghisi, M., \& Gobbino, M. (2009). Spectral gap global solutions for degenerate Kirchhoff equations. Nonlinear Analysis, 71, 4115-4124. https://doi.org/10.1016/j.na.2009.02.090

Guoguang Lin, Yunlong Gao, \& Yuting Sun. (2017). On Local Existence and Blow-Up of Solutions for Nonlinear Wave Equations of Higher-Order Kirchhoff Type with Strong Dissipation. International Journal of Modern Nonlinear Theory and Application, 6, 11-25. https://doi.org/10.4236/ijmnta.2017.61002

HARAUX, A, \& ZUAZUA, E. (1988). Decay estimates for some semilinear damped hyperbolic problems. Arch. Rational Mech. Anal., 100(2), 191-206. https://doi.org/10.1016/j.jde.2011.08.022

Igor Chueshov. (2012). Longtime dynamics of Kirchhoff wave models with strong nonlinear damping. J. Differential Equations, 252, 1229-1262. https://doi.org/10.1016/j.jde.2011.08.022

Ke, Li. (2017). A Gronwall-type lemma with parameter and its application to Kirchhoff type nonlinear wave equation. Journal of Mathematical Analysis and Applications, 447, 683-704. https://doi.org/10.1016/j.jmaa.2016.10.017

Kirchhoff, G., \& Vorlesungen über, Mechanik. (1883). Teubner, Leipzig.

KOPÁC̆KOVÁ, M. (1989). Remarks on bounded solutions of a semilinear dissipative hyperbolic equation. Conment. Math. Univ. Carolin., 30(4), 713-719. 
Kosuke, Ono. (1997). On Global Existence, Asymptotic Stability and Blowing Up of Solutions for Some Degenerate Non-linear Wave Equations of Kirchhoff Type with a Strong Dissipation. Mathematical Methods in the Applied Sciences, 20, 151-177. https://doi.org/10.1002/(SICI)1099-1476(19970125)20:2;151::AID-MMA851;3.0.CO;2-0

Li, Y. (2011). The Asymptotic Behavior of Solutions for a Nonlinear Higher Order Kirchhoff Type Equation. Journal of Southwest China Normal University, 36, 24-27.

Lin, G. G. (2011). Nonlinear evolution equation. Yunnan University Press.

Nguyen, H. D. (2014). A Digital Binomial Theorem. Mathematics, 1-5.

Robert, A. A., \& Fournier, J. J. F. (2003). Sobolev spaces. Department of Mathematics The University of British Columbia Vancouver, Canada.

Teman, R. (1998). Infinite Dimensional Dynamics Systems in Mechanics and Physics. Springer, New York.

Wu, J. Z., \& Lin, G. G. (2009). The global attractor of the Bossinesq equation with damping term and its dimension estimation. Journal of Yunnan University, 31, 335-340.

Xiaoming, Fan, \& Shengfan, Zhou. (2004). Kernel sections for non-autonomous strongly damped wave equations of non-degenerate Kirchhoff-type. Applied Mathematics and Computation, 158, 253-266. https://doi.org/10.1016/j.amc.2003.08.147

Xueli, Song, \& Yanren, Hou. (2015). Uniform attractors for three-dimensional Navier-Stokes equations with nonlinear damping. Journal of Mathematical Analysis and Applications, 422, 337-351. https://doi.org/10.1016/j.aml.2014.02.014

Yang, Z. J. (2010). Finite-dimensional attractors for the Kirchhoff models. J. Math. Phys, 51.

Yang, Z. J., Ding, P. Y., \& Liu, Z. M. (2014). Global attractor for the Kirchhoff type equations with strong nonlinear damping and supercritical nonlinearity. Applied Mathematics Letters, 33, 12-17. https://doi.org/10.1016/j.aml.2014.02.014

Yang, Z. J., Ding, P. Y. , \& Li, L. (2016). Longtime dynamics of the Kirchhoff equations with fractional damping and supercritical nonlinearity. Journal of Mathematical Analysis Application, 442, 485-510. https://doi.org/10.1016/j.jmaa.2016.04.079

Yaojun, Ye. (2013). Global existence and energy decay estimate of solutions for a higher-order Kirchhoff type equation with damping and source term. Nonlinear Analysis, Real World Applications, 14, 2059-2067. https://doi.org/10.1016/j.nonrwa.2013.03.001

Yunlong Gao, Yuting Sun, \& Guoguang Lin. (2016). The Global Attractor and Their Hausdorff and Fractal Dimensions Estimation for the Higher-Order Nonlinear Kirchhoff-Type Equation with Strong Linear Damping. International Journal of Modern Nonlinear Theory and Application, 5, 185-202. https://doi.org/10.4236/ijmnta.2016.54018

Zhijian Yang, \& Zhiming Liu. (2015). Exponential attractor for the Kirchhoff equations with strong nonlinear damping and supercritical nonlinearity. Applied Mathematics Letters, 46, 350-359. https://doi.org/10.1016/j.jmaa.2015.10.013

Zhang Yan, Pu Zhilin, \& Chen Botao. (2008) Boundedness of the Solution to the Nonlinear Kirchhoff Equation. Journal of Southwest China Normal University, 6, 5-8.

Zhijian Yang, \& Pengyan Ding. (2016). Longtime dynamics of the Kirchhoff equation with strong damping and critical nonlinearity on $R^{N}$. Journal of Mathematical Analysis and Applications, 434, 1826-1851.

https://doi.org/10.1016/j.jmaa.2015.10.013

Zhou, S. (1999). Global attractor for strongly damped nonlinear wave equations, 6, 451-470.

\section{Copyrights}

Copyright for this article is retained by the author(s), with first publication rights granted to the journal.

This is an open-access article distributed under the terms and conditions of the Creative Commons Attribution license (http://creativecommons.org/licenses/by/4.0/). 